\title{
Weak subordination of multivariate Lévy processes and variance generalised gamma convolutions
}

\author{
BORIS BUCHMANN ${ }^{1}$, KEVIN W. LU² and DILIP B. MADAN ${ }^{3}$ \\ ${ }^{1}$ Research School of Finance, Actuarial Studies \& Statistics, Australian National University, ACT 0200, \\ Australia.E-mail: Boris.Buchmann@anu.edu.au \\ ${ }^{2}$ Mathematical Sciences Institute, Australian National University, ACT 0200, Australia. \\ E-mail:kevin.lu@anu.edu.au \\ ${ }^{3}$ Robert H. Smith School of Business, University of Maryland, College Park, MD 20742, USA. \\ E-mail:dbm@rhsmith.umd.edu
}

Subordinating a multivariate Lévy process, the subordinate, with a univariate subordinator gives rise to a pathwise construction of a new Lévy process, provided the subordinator and the subordinate are independent processes. The variance-gamma model in finance was generated accordingly from a Brownian motion and a gamma process. Alternatively, multivariate subordination can be used to create Lévy processes, but this requires the subordinate to have independent components. In this paper, we show that there exists another operation acting on pairs $(T, X)$ of Lévy processes which creates a Lévy process $X \odot T$. Here, $T$ is a subordinator, but $X$ is an arbitrary Lévy process with possibly dependent components. We show that this method is an extension of both univariate and multivariate subordination and provide two applications. We illustrate our methods giving a weak formulation of the variance- $\boldsymbol{\alpha}$-gamma process that exhibits a wider range of dependence than using traditional subordination. Also, the variance generalised gamma convolution class of Lévy processes formed by subordinating Brownian motion with Thorin subordinators is further extended using weak subordination.

Keywords: Brownian motion; gamma process; generalised gamma convolutions; Lévy process; marked point process; subordination; Thorin measure; variance gamma; variance-alpha-gamma

\section{Introduction}

The subordination of Lévy processes has many important applications. In mathematical finance, for instance, it acts as a time change that models the flow of information, measuring time in volume of trade as opposed to real time. This idea was initiated by [16] who introduced the variance-gamma process for modelling stock prices, where the subordinate is Brownian motion and the subordinator is a gamma process. Multivariate subordination can be applied to model dependence across multivariate Lévy processes, where the components may have common and/or idiosyncratic time changes. We refer the reader to [7] for a thorough discussion of traditional subordination and its applications. 
Let $T=\left(T_{1}, \ldots, T_{n}\right)$ be an $n$-dimensional subordinator, and $X=\left(X_{1}, \ldots, X_{n}\right)$ be another $n$-dimensional Lévy process called the subordinate. Subordination is the operation that produces the $n$-dimensional process $X \circ T$ defined by

$$
(X \circ T)(t):=\left(X_{1}\left(T_{1}(t)\right), \ldots, X_{n}\left(T_{n}(t)\right)\right), \quad t \geq 0 .
$$

If $T$ and $X$ are independent, then there are two important special cases where $X \circ T$ is again a Lévy process:

- Traditional Bochner subordination, where $T_{1}=T_{2}=\cdots=T_{n}$ are indistinguishable $[4,20$, $21,26]$.

- $T$ is multivariate but $X$ has independent components $X_{1}, \ldots, X_{n}$ [3].

Thus, for strictly multivariate subordination, that is $n \geq 2$, while $T$ does not have indistinguishable components, we have to restrict the class of admissible subordinates $X$ to Lévy processes with independent components, which is, as we show in Proposition 3.9 below, in some cases necessary if we are to stay in the class of Lévy processes.

In the present paper, we show that there exists an operation that extends the traditional notion of subordination by assigning the distribution of a Lévy process $X \odot T$ to the pair $(T, X)$ of Lévy processes. The weakly subordinated process $X \odot T$ is a general Lévy process, it inherits jumps from the multivariate subordinator $T$, which resembles subordination, and our new operation reduces to subordination when the components of $X$ are independent or the components of $T$ are indistinguishable.

The remaining parts of the paper are organised as follows. In Section 2, we define weak subordination, show its existence and that there is a stronger pathwise interpretation, based on marked point processes of jumps, which we call semi-strong subordination. We review some properties of gamma and variance-gamma processes. Further, we introduce a weakly subordinated version of the variance- $\boldsymbol{\alpha}$-gamma process as an extension of the strongly subordinated version in [23]. We develop this new class in a number of remarks throughout the paper to illustrate our machinery.

Section 3 contains a number of results concerning the relation between traditional subordination and weak subordination. In particular, we show that weak subordination extends traditional subordination and is consistent with projecting to marginal distributions, like traditional subordination. However, there are also differences between both notions. To highlight these, we provide formulae for the first and second moments and covariances for weakly subordinated processes.

In Section 4, we exemplify the unifying nature of weak subordination as illustrated using variance generalised gamma convolutions. In [11], a class of processes was introduced by subordinating $n$-dimensional Brownian motion with univariate subordinators taken from Thorin's [24, 25] class of generalised gamma convolutions $(G G C)$. This class has been coined $V G G^{n, 1}$ in [7] who complemented it with their $V G G^{n, n}$-class, obtained by subordinating $n$-dimensional Brownian motion with independent components with $n$-dimensionional $G G C$-subordinators. Using weak subordination, we introduce a weak $V G G^{n}$-class of Lévy processes as a natural superclass of the $V G G^{n, 1}$ and $V G G^{n, n}$-classes. Unifying the results in [7], we provide formulae for the associated characteristic function and Lévy measure.

Section 5 contains technical proofs. 


\section{Main results}

Let $\mathbb{R}^{n}$ be $n$-dimensional Euclidean space whose elements are row vectors $\mathbf{x}=\left(x_{1}, \ldots, x_{n}\right)$, with canonical basis $\left\{\mathbf{e}_{k}: 1 \leq k \leq n\right\}$, and let $\mathbf{e}:=(1, \ldots, 1) \in \mathbb{R}^{n}$. Let $\mathbf{x}^{\prime}, \Sigma^{\prime}$ denote the transpose of a vector $\mathbf{x}$ and a matrix $\Sigma$, respectively. Let $\langle\mathbf{x}, \mathbf{y}\rangle=\mathbf{x y}^{\prime}$ denote the Euclidean product with Euclidean norm $\|\mathbf{x}\|^{2}=\langle\mathbf{x}, \mathbf{x}\rangle=\mathbf{x} \mathbf{x}^{\prime}$, and set $\langle\mathbf{x}, \mathbf{y}\rangle_{\Sigma}:=\mathbf{x} \Sigma \mathbf{y}^{\prime}$ and $\|\mathbf{x}\|_{\Sigma}^{2}:=\langle\mathbf{x}, \mathbf{x}\rangle_{\Sigma}$ for $\mathbf{x}, \mathbf{y} \in \mathbb{R}^{n}$ and $\Sigma \in \mathbb{R}^{n \times n}$. Let $\mathbb{D}:=\left\{\mathbf{x} \in \mathbb{R}^{n}:\|\mathbf{x}\| \leq 1\right\}$ be the Euclidean unit ball centred at the origin. If $A \subseteq \mathbb{R}^{n}$, set $A_{*}:=A \backslash\{\boldsymbol{0}\}$ and let $\mathbf{1}_{A}(\omega)=\boldsymbol{\delta}_{\omega}(A)$ denote the indicator function and the Dirac measure, respectively. Further, $I:[0, \infty)^{n} \rightarrow[0, \infty)^{n}$ and $\ln : \mathbb{C} \backslash(-\infty, 0] \rightarrow \mathbb{C}$ denote the identity function and the principal branch of the logarithm, respectively. The decomposition of an extended real number $x \in[-\infty, \infty]$ into its positive and negative parts is denoted by $x=x^{+}-x^{-}$, where $x^{+}=x \vee 0$ and $x^{-}=(-x)^{+}=-(x \wedge 0)$.

If $\varnothing \neq J \subseteq\{1, \ldots, n\}$, introduce the associated projection $\pi_{J}: \mathbb{R}^{n} \rightarrow \mathbb{R}^{n}$ by $\boldsymbol{\pi}_{J}(\mathbf{x}):=\mathbf{x} \boldsymbol{\pi}_{J}:=$ $\sum_{j \in J} x_{j} \mathbf{e}_{j}$. If $\mathcal{V}$ is a Borel measure on $\mathbb{R}^{n}$, then so is the image (push forward) measure $\mathcal{V}_{J}:=$ $\mathcal{V} \circ \pi_{J}^{-1}$. If $\mathcal{X}$ is a Borel measure on $\mathbb{R}_{*}^{n}$, then so is $\mathcal{X}_{J}$, which is constructed in the usual way: first extend $\mathcal{X}$ to a Borel measure $\mathcal{V}$ on $\mathbb{R}^{n}$ by setting $\mathcal{V}(\{\mathbf{0}\}):=0$, second let $\mathcal{X}_{J}$ be the restriction of $\mathcal{V}_{J}$ to $\mathbb{R}_{*}^{n}$. If $J=\varnothing$, we employ the conventions $\boldsymbol{\pi}_{\varnothing} \equiv \mathbf{0}, \mathcal{V}_{\varnothing} \equiv 0$ and $\mathcal{X}_{\varnothing} \equiv 0$.

The reader is referred to the monographs $[1,5,21]$ for necessary material on Lévy processes. Particularly, the law of a Lévy process $X=\left(X_{1}, \ldots, X_{n}\right)=(X(t))_{t \geq 0}$ is determined by its characteristic function

$$
\Phi_{X(t)}(\boldsymbol{\theta}):=\mathbb{E} \exp \{\mathrm{i}\langle\boldsymbol{\theta}, X(t)\rangle\}=\exp \left\{t \Psi_{X}(\boldsymbol{\theta})\right\}, \quad t \geq 0,
$$

with Lévy exponent $\Psi_{X}=\Psi$ where, $\boldsymbol{\theta} \in \mathbb{R}^{n}$,

$$
\Psi(\boldsymbol{\theta})=\mathrm{i}\langle\boldsymbol{\mu}, \boldsymbol{\theta}\rangle-\frac{1}{2}\|\boldsymbol{\theta}\|_{\Sigma}^{2}+\int_{\mathbb{R}_{*}^{n}}\left(e^{\mathrm{i}\langle\boldsymbol{\theta}, \mathbf{x}\rangle}-1-\mathrm{i}\langle\boldsymbol{\theta}, \mathbf{x}\rangle \mathbf{1}_{\mathbb{D}}(\mathbf{x})\right) \mathcal{X}(\mathrm{d} \mathbf{x}) .
$$

Here, $\boldsymbol{\mu}=\left(\mu, \ldots, \mu_{n}\right) \in \mathbb{R}^{n}$ is a row vector, $\Sigma=\left(\Sigma_{k l}\right) \in \mathbb{R}^{n \times n}$ is a covariance matrix, and $\mathcal{X}$ is a nonnegative Borel measure on $\mathbb{R}_{*}^{n}$ satisfying

$$
\int_{\mathbb{R}_{*}^{n}}\|\mathbf{x}\|^{2} \wedge 1 \mathcal{X}(\mathrm{d} \mathbf{x})<\infty .
$$

We write $X \sim L^{n}(\boldsymbol{\mu}, \Sigma, \mathcal{X})$, provided $X$ is an $n$-dimensional Lévy process with canonical triplet $(\mu, \Sigma, \mathcal{X})$. Throughout, $B=\left(B_{1}, \ldots, B_{n}\right) \sim B M^{n}(\mu, \Sigma):=L^{n}(\mu, \Sigma, 0)$ refers to an $n$-dimensional Brownian motion $B$ with linear drift $\mathbb{E}[B(t)]=\mu t$ and covariance matrix $\operatorname{Cov}(B(t))=t \Sigma, t \geq 0$.

We write $X \sim F V^{n}(\mathbf{d}, \mathcal{X})$ with $\mathbf{d}:=\boldsymbol{\mu}-\int_{\mathbb{D}_{*}} \mathbf{x} \mathcal{X}(\mathrm{d} \mathbf{x}) \in \mathbb{R}^{n}$ denoting the drift of $X$, provided the paths of $X$ are of (locally) finite variation, equivalently, $\Sigma=0$ and

$$
\int_{\mathbb{D}_{*}}\|\mathbf{x}\| \mathcal{X}(\mathrm{d} \mathbf{x})<\infty .
$$

Particularly, $T=\left(T_{1}, \ldots, T_{n}\right) \sim S^{n}(\mathbf{d}, \mathcal{T})$ refers to an $n$-dimensional subordinator, that is a Lévy process with nondecreasing components with drift $\mathbf{d} \in[0, \infty)^{n}$ and Lévy measure $\mathcal{T}$. 
Next, we revise some properties of gamma and variance-gamma processes. Notation is borrowed from [7].

Gamma subordinator. If $a, b>0$, then a subordinator $G$ is a gamma subordinator if and only if its marginal $G(t) \sim \Gamma(a t, b), t \geq 0$ is gamma distributed with shape parameter $a t$ and rate parameter $b$. A drift-less subordinator $G$ with Lévy measure $\mathcal{G}_{a, b}$ is a gamma subordinator with parameters $a, b$, provided its Lévy measure satisfies $\mathcal{G}_{a, b}(\mathrm{~d} g)=\mathbf{1}_{(0, \infty)}(g) a e^{-b g} \mathrm{~d} g / g$, in short, $G \sim \Gamma_{S}(a, b)=S^{1}\left(0, \mathcal{G}_{a, b}\right)$. If $G \sim \Gamma_{S}(a, b)$ and $\lambda>-b$, its Laplace exponent is $-\ln \mathbb{E}[\exp \{-\lambda G(t)\}]=a t \ln \{(b+\lambda) / b\}$.

If $a=b$, we refer to $G$ as a standard gamma subordinator, in short, $G \sim \Gamma_{S}(b):=\Gamma_{S}(b, b)$ and its Lévy measure is denoted by $\mathcal{G}_{b}$. A gamma subordinator $G$ is a standard gamma subordinator if and only if $\mathbb{E}[G(1)]=1$.

Variance-gamma process. Let $b>0, \mu \in \mathbb{R}^{n}$ and $\Sigma \in \mathbb{R}^{n \times n}$ be a covariance matrix. For a Brownian motion $B \sim B M^{n}(\mu, \Sigma)$ independent of a gamma subordinator $G \sim \Gamma_{S}(b)$, we call $V$ a variance-gamma $\left(V G^{n}\right)$ process [16] with parameters $b, \mu, \Sigma$, if

$$
V \stackrel{D}{=} B \circ(G \mathbf{e}) \sim V G^{n}(b, \mu, \Sigma)=B M^{n}(\boldsymbol{\mu}, \Sigma) \circ\left(\Gamma_{S}(b) \mathbf{e}\right) .
$$

An $n$-dimensional Lévy process $V$ is a $V G^{n}(b, \mu, \Sigma)$-process if and only if its characteristic exponent has the form (see [7], their Formula (2.9))

$$
\Psi_{V}(\boldsymbol{\theta})=-b \ln \left\{\left(b-\mathrm{i}\langle\boldsymbol{\mu}, \boldsymbol{\theta}\rangle+(1 / 2)\|\boldsymbol{\theta}\|_{\Sigma}^{2}\right) / b\right\}, \quad \boldsymbol{\theta} \in \mathbb{R}^{n} .
$$

Alternatively, a drift-less $F V^{n}$-process $X$ is a $V G^{n}(b, \mu, \Sigma)$-process if and only if its Lévy measure satisfies $\mathcal{X}=\mathcal{V}_{b, \mu, \Sigma}$ for some $b>0, \mu \in \mathbb{R}^{n}$, a covariance matrix $\Sigma \in \mathbb{R}^{n \times n}$ and $B \sim$ $B M^{n}(\mu, \Sigma)$, where

$$
\mathcal{V}_{b, \mu, \Sigma}(\mathrm{d} \mathbf{y}):=\int_{(0, \infty)} \mathbb{P}(B(g) \in \mathrm{d} \mathbf{y}) b e^{-b g} \mathrm{~d} g / g .
$$

This follows from the formula of the Lévy measure under univariate subordination (see [21], his Formula (30.8)).

If, in addition, $\Sigma$ is invertible, then $\mathcal{V}_{b, \mu, \Sigma}$ is absolutely continuous with respect to the Lebesgue measure $\mathrm{d} \mathbf{v}$ on $\mathbb{R}_{*}^{n}$, having Lévy density $v_{b, \boldsymbol{\mu}, \Sigma}(\mathbf{v}):=\left(\mathrm{d} \mathcal{V}_{b, \mu, \Sigma} / \mathrm{d} \mathbf{v}\right)(\mathbf{v}), \mathbf{v} \in \mathbb{R}_{*}^{n}$, where (see [7], their Formula (2.11))

$$
v_{b, \boldsymbol{\mu}, \Sigma}(\mathbf{v})=\frac{2 b \exp \left\{\langle\boldsymbol{\mu}, \mathbf{v}\rangle_{\Sigma^{-1}}\right\}}{(2 \pi)^{n / 2}|\Sigma|^{1 / 2}\|\mathbf{v}\|_{\Sigma^{-1}}^{n}} \mathfrak{K}_{n / 2}\left\{\left(2 b+\|\boldsymbol{\mu}\|_{\Sigma^{-1}}^{2}\right)^{1 / 2}\|\mathbf{v}\|_{\Sigma^{-1}}\right\},
$$

$\mathfrak{K}_{\rho}(r):=r^{\rho} K_{\rho}(r), \rho \geq 0, r>0$, and $K_{\rho}$ is a modified Bessel function of the second kind (see [7] and [10], their Equation (2.12) and their Equation (3.471)-9, respectively).

Multivariate time parameter. If $X, Y \sim L^{n}$ are independent $n$-dimensional Lévy processes, then the $2 n$-dimensional process $Z=(X, Y) \sim L^{2 n}(\mathbf{m}, \Sigma, \mathcal{Z})$ is a Lévy process in $\mathbb{R}^{2 n}$, for some $\mathbf{m} \in \mathbb{R}^{2 n}, \Sigma \in \mathbb{R}^{2 n \times 2 n}$, and Lévy measure $\mathcal{Z}$ on $\mathbb{R}_{*}^{2 n}$. Our notation extends from $\mathbb{R}^{n}$ to $\mathbb{R}^{2 n}$ in a canonical way; in particular, $\|\cdot\|$ and $\mathbb{D}$ may refer to the Euclidean norm and the Euclidean unit ball in $\mathbb{R}^{n}$ as well as in $\mathbb{R}^{2 n}$, respectively. 
As a first step, we evaluate an $n$-dimensional Lévy process $X=\left(X_{1}, \ldots, X_{n}\right)$, indexed by univariate time $t$, at multivariate time points $\mathbf{t}=\left(t_{1}, \ldots, t_{n}\right) \in[0, \infty)^{n}$. The result is an infinitely divisible row vector $X(\mathbf{t})=\left(X_{1}\left(t_{1}\right), \ldots, X_{n}\left(t_{n}\right)\right)$. To provide formulae for the associated characteristics, we introduce an operation $\diamond$ as an outer product.

For $\mathbf{t}=\left(t_{1}, \ldots, t_{n}\right) \in[0, \infty)^{n}, \boldsymbol{\mu}=\left(\mu_{1}, \ldots \mu_{n}\right) \in \mathbb{R}^{n}$ and $\Sigma=\left(\Sigma_{k l}\right) \in \mathbb{R}^{n \times n}$, introduce $\mathbf{t} \diamond \boldsymbol{\mu} \in$ $\mathbb{R}^{n}$ and $\mathbf{t} \diamond \Sigma=\left(\mathbf{t} \diamond \Sigma_{k l}\right) \in \mathbb{R}^{n \times n}$ by

$$
\mathbf{t} \diamond \boldsymbol{\mu}:=\left(t_{1} \mu_{1}, \ldots, t_{n} \mu_{n}\right), \quad(\mathbf{t} \diamond \Sigma)_{k l}:=\Sigma_{k l}\left(t_{k} \wedge t_{l}\right), \quad 1 \leq k, l \leq n .
$$

Choose an ordering $t_{(1)} \leq \ldots \leq t_{(n)}$ of the components with associated permutation $\langle(1), \ldots,(n)\rangle$ and spacings $\Delta t_{(k)}:=t_{(k)}-t_{(k-1)}$ for $1 \leq k \leq n, t_{(0)}:=0$. If $\mathcal{X}$ is a Lévy measure, thus a Borel measure on $\mathbb{R}^{n}$ satisfying (2.2), so is $\mathbf{t} \diamond \overline{\mathcal{X}}$, defined by

$$
\mathbf{t} \diamond \mathcal{X}:=\sum_{k=1}^{n} \Delta t_{(k)} \mathcal{X}_{\{(k), \ldots,(n)\}} .
$$

We introduce $\mathbf{c}(\mathbf{t}, \mathcal{X}) \in \mathbb{R}^{n}$ by setting

$$
\mathbf{c}:=\mathbf{c}(\mathbf{t}, \mathcal{X}):=\sum_{k=2}^{n} \Delta t_{(k)} \int_{\mathbb{D}^{C}} \boldsymbol{\pi}_{\{(k), \ldots,(n)\}}(\mathbf{x}) \mathbf{1}_{\mathbb{D}}\left(\boldsymbol{\pi}_{\{(k), \ldots,(n)\}}(\mathbf{x})\right) \mathcal{X}(\mathrm{d} \mathbf{x}) .
$$

As (2.2) is satisfied for a Lévy measure $\mathcal{X}, \mathbf{c}(\mathbf{t}, \mathcal{X})$ is a well-defined $n$-dimensional row vector, and it acts as a compensation term.

We provide formulae for the characteristics of $X(\mathbf{t})$ (see Section 5.1 for a proof).

Proposition 2.1. For $\mathbf{t}=\left(t_{1}, \ldots, t_{n}\right) \in[0, \infty)^{n}$ and $X \sim L^{n}(\mu, \Sigma, \mathcal{X})$ with $\Psi$ as in (2.1), the vector $X(\mathbf{t})=\left(X_{1}\left(t_{1}\right), \ldots, X_{n}\left(t_{n}\right)\right) \in \mathbb{R}^{n}$ is infinitely divisible with $\Phi_{X(\mathbf{t})}(\boldsymbol{\theta})=\mathbb{E} \exp (\mathrm{i}\langle\boldsymbol{\theta}$, $X(\mathbf{t})\rangle)=\exp ((\mathbf{t} \diamond \Psi)(\boldsymbol{\theta})), \boldsymbol{\theta} \in \mathbb{R}^{n}$, where

$$
\begin{aligned}
(\mathbf{t} \diamond \Psi)(\boldsymbol{\theta}):= & \sum_{k=1}^{n} \Delta t_{(k)} \Psi\left(\boldsymbol{\pi}_{\{(k), \ldots,(n)\}}(\boldsymbol{\theta})\right) \\
= & \mathrm{i}\langle\mathbf{t} \diamond \boldsymbol{\mu}+\mathbf{c}, \boldsymbol{\theta}\rangle-\frac{1}{2}\|\boldsymbol{\theta}\|_{\mathbf{t} \diamond \Sigma}^{2} \\
& +\int_{\mathbb{R}_{*}^{n}}\left(e^{\mathrm{i}\langle\boldsymbol{\theta}, \mathbf{x}\rangle}-1-\mathrm{i}\langle\boldsymbol{\theta}, \mathbf{x}\rangle \mathbf{1}_{\mathbb{D}}(\mathbf{x})\right) \mathbf{t} \diamond \mathcal{X}(\mathrm{d} \mathbf{x}) .
\end{aligned}
$$

Remark 2.1. If $B$ is a standard Brownian motion and $I$ is the identity function, then $(I, 2 I)$ is a subordinator and $(B, B)$ is a Lévy process, but $(B, B) \circ(I, 2 I)$ is not a Lévy process, as follows from Proposition 3.9(iii) below. Though it is a Gaussian process, $(B, B) \circ(I, 2 I)$ is not a Brownian motion.

Weak subordination. If $T \sim S^{n}(\mathbf{d}, \mathcal{T})$ is a subordinator, then we may write $T=I \mathbf{d}+S$, where $S \sim S^{n}(\mathbf{0}, \mathcal{T})$ is a pure jump subordinator and $I \mathbf{d}$ is a deterministic subordinator. Suppose $X \sim$ 
$L^{n}(\boldsymbol{\mu}, \Sigma, \mathcal{X})$ is the candidate for a subordinate. If $Y$ is another Lévy process with $Y \sim L^{n}(\mathbf{d} \diamond$ $\boldsymbol{\mu}+\mathbf{c}, \mathbf{d} \diamond \Sigma, \mathbf{d} \diamond \mathcal{X})$, we get from Proposition 2.1 that $X(t \mathbf{d}) \stackrel{D}{=} Y(t)$ for all fixed $t \geq 0$. Any other Lévy process with this property must have the same characteristics, and in the case of deterministic subordination the law of weak subordination is determined.

On the other hand, if $\mathbf{d}=\mathbf{0}$ and $T=S$, we may perceive the subordinated process as a $2 n$ dimensional Lévy process $Z=(S, Y)$ in time-space $[0, \infty)^{n} \times \mathbb{R}^{n}$, and the jumps of $Y$ should have conditional laws $(\Delta Y \mid \Delta T=\mathbf{t}) \sim \mathbb{P}(X(\mathbf{t}) \in \cdot)$. This notion is consistent with traditional subordination (see [3] and [21], their Formula (3.12) and his Formula (30.8), respectively) as illustrated by (2.5) in the context of $V G^{n}$-processes. Equivalently, the jumps of the joint process $Z$ form a marked point process, with marks in time-space $[0, \infty)_{*}^{n} \times \mathbb{R}_{*}^{n}$ determined, conditionally independently, based on the points of a Poisson point process in time-time $[0, \infty) \times[0, \infty)_{*}^{n}$, with intensity measure $\mathrm{d} t \otimes \mathcal{T}$. Summing up those jumps along $t \geq 0$, possibly with a compensation term, generates a pure-jump Lévy process $Z$ with values in time-space $[0, \infty)^{n} \times \mathbb{R}^{n}$. Using pure-jump subordinators, the law of weak subordination is thus determined in time-space.

Traditional subordination is consistent with the superposition of independent subordinators such as $T=I \mathbf{d}+S$, and the law of strongly subordinated processes, when defined as Lévy processes, is determined by convolution (see [7], their Proposition 4.1). Together with Proposition 3.1, this imposes a final and determining constraint on the law of weak subordination.

We are now prepared to introduce subordination in the weak and semi-strong senses.

Definition 2.1. Let $X \sim L^{n}(\boldsymbol{\mu}, \Sigma, \mathcal{X})$ and $T \sim S^{n}(\mathbf{d}, \mathcal{T})$. A process $Z$ is called a subordinator $T$ subordinating $X$ in the weak sense, meaning that $Z \stackrel{D}{=}(T, X \odot T)$, whenever $Z=\left(Z_{1}, Z_{2}\right) \sim$ $L^{2 n}(\mathbf{m}, \Theta, \mathcal{Z})$ is a Lévy process with the characteristics determined by $\mathbf{m}=\left(\mathbf{m}_{1}, \mathbf{m}_{2}\right), \mathbf{m}_{1}, \mathbf{m}_{2} \in$ $\mathbb{R}^{n}$,

$$
\begin{aligned}
& \mathbf{m}_{1}=\mathbf{d}+\int_{[0, \infty)_{*}^{n}} \mathbf{t} \mathbb{P}((\mathbf{t}, X(\mathbf{t})) \in \mathbb{D}) \mathcal{T}(\mathrm{d} \mathbf{t}), \\
& \mathbf{m}_{2}=\mathbf{c}(\mathbf{d}, \mathcal{X})+\mathbf{d} \diamond \boldsymbol{\mu}+\int_{[0, \infty)_{*}^{n}} \mathbb{E}\left[X(\mathbf{t}) \mathbf{1}_{\mathbb{D}}(\mathbf{t}, X(\mathbf{t}))\right] \mathcal{T}(\mathrm{d} \mathbf{t}), \\
& \Theta=\left(\begin{array}{cc}
\mathbf{0} & \mathbf{0} \\
\mathbf{0} & \mathbf{d} \diamond \Sigma
\end{array}\right), \\
& \mathcal{Z}(\mathrm{d} \mathbf{t}, \mathrm{d} \mathbf{x})=\left(\boldsymbol{\delta}_{\mathbf{0}} \otimes(\mathbf{d} \diamond \mathcal{X})\right)(\mathrm{d} \mathbf{t}, \mathrm{d} \mathbf{x})+\mathbf{1}_{[0, \infty)_{*}^{n} \times \mathbb{R}^{n}} \mathbb{P}(X(\mathbf{t}) \in \mathrm{d} \mathbf{x}) \mathcal{T}(\mathrm{d} \mathbf{t}) .
\end{aligned}
$$

We call $Z=\left(Z_{1}, Z_{2}\right)$ a subordinator $T$ subordinating $X$ in the semi-strong sense, whenever, simultaneously, $Z_{1}=T$ are indistinguishable and $Z \stackrel{D}{=}(T, X \odot T)$.

Such a process $Z$ exists and is a Lévy process whenever the specifying characteristics are as in (2.12)-(2.15). The main difficulty is to show that $\mathbb{P}(X(\mathbf{t}) \in \mathrm{d} \mathbf{x}) \mathcal{T}(\mathrm{d} \mathbf{t})$ is a Lévy measure. Semi-strong subordination is then always possible on augmented probability spaces, and it relies on marking the point process associated to the jumps of $T$ (see Section 5.2 for a proof).

Theorem 2.1. Let $X$ and $T$ be as in Definition 2.1. 
(i) There exists a Lévy process $Z=\left(Z_{1}, Z_{2}\right) \sim L^{2 n}(\mathbf{m}, \Theta, \mathcal{Z})$ with $\mathbf{m}, \Theta, \mathcal{Z}$ as specified in (2.12)-(2.15).

(ii) On an augmentation of the probability space carrying $T$, there exists an $n$-dimensional Lévy process $Z_{2}$ such that $\left(T, Z_{2}\right)$ is a subordinator $T$ subordinating $X$ in the semi-strong sense.

(iii) If both $\mathbf{d}=\mathbf{0}$ and $\int_{[0,1]_{*}^{n}}\|\mathbf{t}\|^{1 / 2} \mathcal{T}(\mathrm{d} \mathbf{t})<\infty$ hold, then $Z$ is a drift-less $F V^{2 n}$-process.

Remark 2.2. Let $B, B^{*}$ be independent standard Brownian motions, and $I$ be the identity function. $(I, 2 I)$ is a subordinator, and $(B, B)$ is a Lévy process, but $(B, B) \circ(I, 2 I)$ is not (see Remark 2.1). As easily verified from (2.12)-(2.15), the process $\left(I, 2 I, B, B+B^{*}\right)$ is the subordinator $(I, 2 I)$ subordinating $(B, B)$ in the semi-strong sense.

Remark 2.3. Recall $\mathbf{e}=(1, \ldots, 1) \in \mathbb{R}^{n}$. Let $G \sim \Gamma_{S}(b)=S^{1}\left(0, \mathcal{G}_{b}\right)$. In the traditional notion of $V G^{n}, G$ is the sole time change for all the components of an $n$-dimensional Brownian motion. Consistent with our notion of multivariate subordination, we replace $G$ with $G \mathbf{e}$. Note $G \mathbf{e}=$ $(G, \ldots, G)$ is an $n$-dimensional drift-less subordinator with indistinguishable components and $G \mathbf{e} \sim S^{n}\left(\mathbf{0}, \mathcal{G}_{b} \circ(I \mathbf{e})^{-1}\right)$.

Remark 2.4. The $V G^{n}$-process in [16] uses $n$-dimensional Brownian motion as its subordinate and a univariate standard gamma process as its subordinator. The $V G^{n}$-model gives a restrictive dependence structure, where components cannot have idiosyncratic time changes and must have equal kurtosis. These last two deficiencies have been addressed by Luciano and Semeraro's [14, 23 ] variance- $\boldsymbol{\alpha}$-gamma ( $V \boldsymbol{\alpha} G$ ) process by the use of an $\boldsymbol{\alpha}$-gamma subordinator.

$\boldsymbol{\alpha}$-gamma subordinator. Assume $n \geq 2$. Let $a, b>0, \boldsymbol{\alpha}=\left(\alpha_{1}, \ldots, \alpha_{n}\right) \in(0, \infty)^{n}$ such that $b>a \alpha_{k}$ for $k=1, \ldots, n$. Introduce $\beta_{k}:=\left(b-a \alpha_{k}\right) / \alpha_{k}$, and let $G_{0}, \ldots, G_{n}$ be independent gamma subordinators such that $G_{0} \sim \Gamma_{S}(a, b), G_{k} \sim \Gamma_{S}\left(\beta_{k}, b / \alpha_{k}\right), 1 \leq k \leq n$.

We refer to an $n$-dimensional subordinator $T \sim \boldsymbol{\alpha} G^{n}(a, b, \boldsymbol{\alpha})$, as an $\boldsymbol{\alpha}$-gamma $(\boldsymbol{\alpha} G)$ subordinator [23], provided

$$
T=\left(T_{1}, \ldots, T_{n}\right) \stackrel{D}{=} G_{0} \boldsymbol{\alpha}+\left(G_{1}, \ldots, G_{n}\right) .
$$

As perceived in [23], the components of $T$ are univariate standard gamma subordinators $T_{k} \sim$ $\Gamma_{S}\left(b / \alpha_{k}\right), 1 \leq k \leq n$. Further, an $n$-dimensional drift-less subordinator $T$ with Lévy measure $\mathcal{T}$ is an $\boldsymbol{\alpha}$-gamma subordinator with parameters $a, b, \boldsymbol{\alpha}$ if and only if, with $\beta_{1}, \ldots, \beta_{n}$ as above,

$$
\mathcal{T}=\int_{(0, \infty)} \boldsymbol{\delta}_{g \alpha} \mathcal{G}_{a, b}(\mathrm{~d} g)+\sum_{k=1}^{n} \boldsymbol{\delta}_{0}^{\otimes(k-1)} \otimes \mathcal{G}_{\beta_{k}, b / \alpha_{k}} \otimes \boldsymbol{\delta}_{0}^{\otimes(n-k)}
$$

Strong variance- $\boldsymbol{\alpha}$-gamma processes. Assume $n \geq 2$. Let $\boldsymbol{\mu}=\left(\mu_{1}, \ldots, \mu_{n}\right) \in \mathbb{R}^{n}$ and $\Sigma=$ $\operatorname{diag}\left(\Sigma_{11}, \ldots, \Sigma_{n n}\right) \in[0, \infty)^{n \times n}$ be a diagonal matrix.

For independent $B$ and $T$, if $Y \stackrel{D}{=} B \circ T$, where $B \sim B M^{n}(\mu, \Sigma)$ is a Brownian motion with independent components and $T \sim \boldsymbol{\alpha} G^{n}(a, b, \boldsymbol{\alpha})$ is an $\boldsymbol{\alpha} G$-subordinator, then we call $Y$ a (strong) variance- $\boldsymbol{\alpha}$-gamma process [23] with parameters $a, b, \boldsymbol{\alpha}, \boldsymbol{\mu}, \Sigma$, in short,

$$
Y \stackrel{D}{=} B \circ T \sim V \boldsymbol{\alpha} G^{n}(a, b, \boldsymbol{\alpha}, \boldsymbol{\mu}, \Sigma)=B M^{n}(\boldsymbol{\mu}, \Sigma) \circ \boldsymbol{\alpha} G_{S}^{n}(a, b, \boldsymbol{\alpha}) .
$$


Remark 2.5. The Brownian motion subordinate must have independent components, which restricts the dependence structure. In our weak formulation of the $V \boldsymbol{\alpha} G$-process, the subordinate is a Brownian motion with possibly correlated components. Our $W V \boldsymbol{\alpha} G$-process has a wider range of dependence structures, while being parsimoniously parametrised, each component has both common and idiosyncratic time changes, it has $V G$-marginals with independent levels of kurtosis, with the jump measure having full support.

Weak variance- $\boldsymbol{\alpha}$-gamma processes. Assume $n \geq 2$. Let $\boldsymbol{\mu}=\left(\mu_{1}, \ldots, \mu_{n}\right) \in \mathbb{R}^{n}$ and $\Sigma=$ $\left(\Sigma_{k l}\right) \in \mathbb{R}^{n \times n}$ be an arbitrary covariance matrix.

Whenever $Y \stackrel{D}{=} B \odot T$, where $B \sim B M^{n}(\boldsymbol{\mu}, \Sigma)$ is Brownian motion, and $T \sim \boldsymbol{\alpha} G^{n}(a, b, \boldsymbol{\alpha})$ is an $\alpha G$-subordinator, then we call $Y$ a weak variance- $\alpha$-gamma process with parameters $a, b, \boldsymbol{\alpha}, \boldsymbol{\mu}, \Sigma$, in short,

$$
Y \stackrel{D}{=} B \odot T \sim W V \boldsymbol{\alpha} G^{n}(a, b, \boldsymbol{\alpha}, \boldsymbol{\mu}, \Sigma)=B M^{n}(\boldsymbol{\mu}, \Sigma) \odot \boldsymbol{\alpha} G_{S}^{n}(a, b, \boldsymbol{\alpha}) .
$$

We derive the joint Lévy measure $\mathcal{Z}$ of the pair $Z=(T, B \odot T)$. Let $N(\mathrm{~d} \mathbf{x} \mid \boldsymbol{\mu}, \Sigma)$ be the normal law with mean $\boldsymbol{\mu}$ and covariance matrix $\Sigma$. As $B(\mathbf{t}) \sim N(\mathrm{~d} \mathbf{x} \mid \mathbf{t} \diamond \boldsymbol{\mu}, \mathbf{t} \diamond \Sigma)$ for fixed $\mathbf{t} \in[0, \infty)_{*}^{n}$, by Proposition 2.1, it follows from (2.15) and (2.17) that for a Borel set $A \subseteq \mathbb{R}_{*}^{2 n}$,

$$
\begin{aligned}
\mathcal{Z}(A)= & \int_{(0, \infty) \times \mathbb{R}^{n}} \mathbf{1}_{A}(g \boldsymbol{\alpha}, \mathbf{x}) N(\mathrm{~d} \mathbf{x} \mid g \boldsymbol{\alpha} \diamond \boldsymbol{\mu}, g \boldsymbol{\alpha} \diamond \Sigma) \mathcal{G}_{a, b}(\mathrm{~d} g) \\
& +\sum_{k=1}^{n} \int_{(0, \infty) \times \mathbb{R}} \mathbf{1}_{A}\left(g \mathbf{e}_{k}, x_{k} \mathbf{e}_{k}\right) N\left(\mathrm{~d} x_{k} \mid g \mu_{k}, g \Sigma_{k k}\right) \mathcal{G}_{\beta_{k}, b / \alpha_{k}}(\mathrm{~d} g) .
\end{aligned}
$$

Formula (2.20) tells us that $T$ and $B \odot T$ jump together. As a result, weakly subordinated Brownian motion resembles the jump behaviour of a subordinated Brownian motion. Like strong $V \boldsymbol{\alpha} G$ processes in [23], $W V \boldsymbol{\alpha} G$-processes jump in two different ways: either the components jump independently of each other together with one of the subordinators $G_{1}, \ldots, G_{n}$, or the components jump together with the subordinator $G_{0}$.

If, in addition, $\Sigma$ is a diagonal matrix, and (2.20) is projected on space we recover the formulae of the strong variance- $\boldsymbol{\alpha}$-gamma process $B \circ T$, as derived in [14] (see their Theorem 1.1).

\section{Properties of weak subordination}

Let $T \sim S^{n}(\mathbf{d}, \mathcal{T})$ and $X \sim L^{n}(\boldsymbol{\mu}, \Sigma, \mathcal{X})$ be candidates for a subordinator and subordinate in the weak or semi-strong subordination of Definition 2.1.

We provide a formula for the characteristic exponent.

Proposition 3.1. $Z \stackrel{D}{=}(T, X \odot T)$ holds in the weak sense if and only if for all $\boldsymbol{\theta}=\left(\boldsymbol{\theta}_{1}, \boldsymbol{\theta}_{2}\right)$ with $\boldsymbol{\theta}_{1}, \boldsymbol{\theta}_{2} \in \mathbb{R}^{n}$, the characteristic exponent of $Z$ is

$$
\Psi_{Z}(\boldsymbol{\theta})=\mathrm{i}\left\langle\mathbf{d}, \boldsymbol{\theta}_{1}\right\rangle+\left(\mathbf{d} \diamond \Psi_{X}\right)\left(\boldsymbol{\theta}_{2}\right)+\int_{[0, \infty)_{*}^{n}}\left(\Phi_{(\mathbf{t}, X(\mathbf{t}))}(\boldsymbol{\theta})-1\right) \mathcal{T}(\mathrm{d} \mathbf{t}) .
$$

Here, $\left(\mathbf{d} \diamond \Psi_{X}\right)\left(\boldsymbol{\theta}_{2}\right)$ is defined as in $(2.11)$, but with $(\boldsymbol{\theta}, \mathbf{t})$ replaced by $\left(\boldsymbol{\theta}_{2}, \mathbf{d}\right)$. 
Proof. Let $\boldsymbol{\theta}=\left(\boldsymbol{\theta}_{1}, \boldsymbol{\theta}_{2}\right)$ with $\boldsymbol{\theta}_{1}, \boldsymbol{\theta}_{2} \in \mathbb{R}^{n}$. Combining (2.3) and Lemma 5.1 below yields $\mathcal{T}$-integrability of $\mathbf{t} \mapsto \mathbb{E} \exp \{\mathbf{i}\langle\boldsymbol{\theta},(\mathbf{t}, X(\mathbf{t}))\rangle\}-1$ with

$$
\begin{aligned}
& \int_{[0, \infty)_{*}^{n} \times \mathbb{R}^{n}}\left(e^{\mathrm{i}\langle\boldsymbol{\theta},(\mathbf{t}, \mathbf{x})\rangle}-1-\mathrm{i}\langle\boldsymbol{\theta},(\mathbf{t}, \mathbf{x})| \mathbf{1}_{\mathbb{D}}(\mathbf{t}, \mathbf{x})\right) \mathbb{P}(X(\mathbf{t}) \in \mathrm{d} \mathbf{x}) \mathcal{T}(\mathrm{d} \mathbf{t}) \\
& =-\mathrm{i} \int_{[0, \infty)_{*}^{n}}\left(\mathbb{E}\left[\left\langle\boldsymbol{\theta}_{2}, X(\mathbf{t})\right\rangle \mathbf{1}_{\mathbb{D}}(\mathbf{t}, X(\mathbf{t}))\right]+\left\langle\boldsymbol{\theta}_{1}, \mathbf{t}\right\rangle \mathbb{P}((\mathbf{t}, X(\mathbf{t})) \in \mathbb{D})\right) \mathcal{T}(\mathrm{d} \mathbf{t}) \\
& \quad+\int_{[0, \infty)_{*}^{n}}\left(\Phi_{(\mathbf{t}, X(\mathbf{t}))}(\boldsymbol{\theta})-1\right) \mathcal{T}(\mathrm{d} \mathbf{t})
\end{aligned}
$$

Plainly, $Z \stackrel{D}{=}(T, X \odot T)$ if and only if $Z$ has characteristic triplet (2.12)-(2.15), and this is true if and only if (3.1) does, as follows from (3.2).

Apart from determining the distribution of the time-and-space projected processes, the next proposition states that, in analogy with traditional subordination [3,21], weak subordination is consistent with projections.

Proposition 3.2. If $Z=\left(Z_{1}, Z_{2}\right)$ is a Lévy process with $n$-dimensional components $Z_{1}$ and $Z_{2}$ such that $Z=\left(Z_{1}, Z_{2}\right) \stackrel{D}{=}(T, X \odot T)$ holds in the weak sense, then we must have $Z_{1} \stackrel{D}{=} T$ as well as $Z_{2} \sim L^{n}\left(\mathbf{m}_{2}, \Theta_{2}, \mathcal{Z}_{2}\right)$ with

$$
\begin{aligned}
\mathbf{m}_{2} & =\mathbf{c}(\mathbf{d}, \mathcal{X})+\mathbf{d} \diamond \boldsymbol{\mu}+\int_{[0, \infty)_{*}^{n}} \mathbb{E}\left[X(\mathbf{t}) \mathbf{1}_{\mathbb{D}}(X(\mathbf{t}))\right] \mathcal{T}(\mathrm{d} \mathbf{t}), \\
\Theta_{2} & =\mathbf{d} \diamond \Sigma \\
\mathcal{Z}_{2}(\mathrm{~d} \mathbf{x}) & =\mathbf{d} \diamond \mathcal{X}(\mathrm{d} \mathbf{x})+\int_{[0, \infty)_{*}^{n}} \mathbb{P}(X(\mathbf{t}) \in \mathrm{d} \mathbf{x}) \mathcal{T}(\mathrm{d} \mathbf{t}) .
\end{aligned}
$$

In addition, if $J \subseteq\{1, \ldots, n\}$, we have $\left(T \pi_{J},(X \odot T) \pi_{J}\right) \stackrel{D}{=}\left(T \pi_{J},\left(X \pi_{J}\right) \odot\left(T \pi_{J}\right)\right)$ and, particularly, $\left(T_{k},(X \odot T)_{k}\right) \stackrel{D}{=}\left(T_{k}, X_{k} \odot T_{k}\right)$ for $1 \leq k \leq n$.

Proof. Let $Z=\left(Z_{1}, Z_{2}\right) \sim L^{2 n}(\mathbf{m}, \Theta, \mathcal{Z})$ with $\mathbf{m}=\left(\mathbf{m}_{1}, \mathbf{m}_{2}\right), \Theta, \mathcal{Z}$ as specified in (2.12)(2.15). For $\boldsymbol{\theta}_{1}, \boldsymbol{\theta}_{2} \in \mathbb{R}^{n}$ it is straightforwardly checked that $\Psi_{Z}\left(\boldsymbol{\theta}_{1}, \mathbf{0}\right)=\Psi_{T}\left(\boldsymbol{\theta}_{1}\right)$ and $\Psi_{Z}\left(\mathbf{0}, \boldsymbol{\theta}_{2}\right)=$ $\Psi_{Z_{2}}\left(\boldsymbol{\theta}_{2}\right)$, giving $Z_{1} \stackrel{D}{=} T$ and $Z_{2} \sim L^{n}$ with characteristics matching those in (3.3)-(3.5).

Without loss of generality, assume $J \neq \varnothing$ and set $\pi:=\pi_{J}$. It suffices to show that, for all $\boldsymbol{\theta}_{1}, \boldsymbol{\theta}_{2} \in \mathbb{R}^{n}$,

$$
\Psi_{(T \pi,(X \odot T) \pi)}\left(\boldsymbol{\theta}_{1}, \boldsymbol{\theta}_{2}\right)=\Psi_{(T \pi,(X \pi) \odot(T \pi))}\left(\boldsymbol{\theta}_{1}, \boldsymbol{\theta}_{2}\right) .
$$

By noting $\Psi_{(T \pi,(X \odot T) \pi)}\left(\boldsymbol{\theta}_{1}, \boldsymbol{\theta}_{2}\right)=\Psi_{(T, X \odot T)}\left(\boldsymbol{\theta}_{1} \boldsymbol{\pi}, \boldsymbol{\theta}_{2} \boldsymbol{\pi}\right)$, the LHS in (3.6) matches the RHS in (3.1), but with $\left(\boldsymbol{\theta}_{1}, \boldsymbol{\theta}_{2}\right)$ replaced with $\left(\boldsymbol{\theta}_{1} \boldsymbol{\pi}, \boldsymbol{\theta}_{2} \boldsymbol{\pi}\right)$. The RHS in (3.6) equals the RHS in (3.1) with $(T, X)$ replaced with $(T \pi, X \pi)$. To prove the identity in (3.6), it thus suffices to compare the three terms occurring on both sides in (3.6), respectively. 
The projected process $T \boldsymbol{\pi}$ is an $n$-dimensional subordinator with drift $\mathbf{d} \boldsymbol{\pi}$ and Lévy measure $\mathcal{T} \circ \boldsymbol{\pi}^{-1}$. Consequently, the first term on both sides in (3.6) are equal as $\left\langle\boldsymbol{\theta}_{1} \boldsymbol{\pi}, \mathbf{d}\right\rangle=\left\langle\boldsymbol{\theta}_{1}, \mathbf{d} \boldsymbol{\pi}\right\rangle$. The second identity, $\mathbf{d} \diamond \Psi_{X}\left(\boldsymbol{\theta}_{2} \boldsymbol{\pi}\right)=(\mathbf{d} \boldsymbol{\pi}) \diamond \Psi_{X \boldsymbol{\pi}}\left(\boldsymbol{\theta}_{2}\right)$, follows from Proposition 2.1 as $\left\langle\boldsymbol{\theta}_{2} \boldsymbol{\pi}, X(\mathbf{d})\right\rangle=$ $\left\langle\boldsymbol{\theta}_{2},(X(\mathbf{d})) \boldsymbol{\pi}\right\rangle=\left\langle\boldsymbol{\theta}_{2},(X \boldsymbol{\pi})(\mathbf{d} \boldsymbol{\pi})\right\rangle$. The third identity follows from the transformation theorem by recalling that $\mathcal{T} \circ \pi^{-1}$ is the Lévy measure of the projected process $T \boldsymbol{\pi}$, and by $(X \boldsymbol{\pi})(\mathbf{t})=$ $(X \boldsymbol{\pi})(\mathbf{t} \boldsymbol{\pi})$ and $\left\langle\left(\boldsymbol{\theta}_{1} \boldsymbol{\pi}, \boldsymbol{\theta}_{2} \boldsymbol{\pi}\right),(\mathbf{t}, X(\mathbf{t}))\right\rangle=\left\langle\left(\boldsymbol{\theta}_{1}, \boldsymbol{\theta}_{2}\right),(\mathbf{t} \boldsymbol{\pi},(X \boldsymbol{\pi})(\mathbf{t} \boldsymbol{\pi})\rangle, \mathbf{t} \in[0, \infty)^{n}\right.$, as they imply the crucial identity

$$
\int_{[0, \infty)_{*}^{n}}\left(\Phi_{(\mathbf{t}, X(\mathbf{t}))}\left(\boldsymbol{\theta}_{1} \boldsymbol{\pi}, \boldsymbol{\theta}_{2} \boldsymbol{\pi}\right)-1\right) \mathcal{T}(\mathrm{d} \mathbf{t})=\int_{[0, \infty)_{*}^{n}}\left(\Phi_{(\mathbf{t},(X \boldsymbol{\pi})(\mathbf{t}))}(\boldsymbol{\theta})-1\right) \mathcal{T} \circ \boldsymbol{\pi}^{-1}(\mathrm{~d} \mathbf{t}) .
$$

Remark 3.1. Weak subordination is consistent with projections to coordinates by Proposition 3.2. Suppose $Y=\left(Y_{1}, \ldots, Y_{n}\right) \stackrel{D}{=} B \odot T \sim W V \boldsymbol{\alpha} G^{n}(a, b, \boldsymbol{\alpha}, \boldsymbol{\mu}, \Sigma)$ in (2.19), where $B=$ $\left(B_{1}, \ldots, B_{n}\right) \sim B M^{n}(\mu, \Sigma)$ and $T=\left(T_{1}, \ldots, T_{n}\right) \sim \alpha G^{n}(a, b, \boldsymbol{\alpha})$. Assume that $B$ and $T$ are independent, then $Y$ has $V G^{1}$-components. Thus, $Y$ has the same marginal distributions as a strong $V \boldsymbol{\alpha} G^{n}(a, b, \boldsymbol{\alpha}, \boldsymbol{\mu}, \Sigma)$-process [23] because

$$
Y_{k} \stackrel{D}{=}(B \odot T)_{k} \stackrel{D}{=} B_{k} \odot T_{k} \stackrel{D}{=} B_{k} \circ T_{k} \sim V G^{1}\left(b / \alpha_{k}, \mu_{k}, \Sigma_{k k}\right), \quad 1 \leq k \leq n .
$$

Weak and semi-strong subordination extends traditional subordination.

Proposition 3.3. Let $T, X$ be independent. If either $T$ has indistinguishable components or $X$ has independent components, then $(T, X \circ T) \stackrel{D}{=}(T, X \odot T)$ in the semi-strong sense.

Proof. We extend $\langle\mathbf{z}, \mathbf{w}\rangle:=\sum_{k=1}^{n} z_{k} w_{k}$ to $\mathbf{z}, \mathbf{w} \in \mathbb{C}^{n}$. We avoid conjugation.

As we assumed $T$ and $X$ to be independent processes, we get from Proposition 2.1 by conditioning on $T$ that, for $\boldsymbol{\theta}=\left(\boldsymbol{\theta}_{1}, \boldsymbol{\theta}_{2}\right), \boldsymbol{\theta}_{1}, \boldsymbol{\theta}_{2} \in \mathbb{R}^{n}$,

$$
\Phi_{(T(1), X(T(1)))}(\boldsymbol{\theta})=\mathbb{E} \exp \left\{\mathrm{i}\left\langle\boldsymbol{\theta}_{1}, T(1)\right\rangle+\left(T(1) \diamond \Psi_{X}\right)\left(\boldsymbol{\theta}_{2}\right)\right\} .
$$

Univariate subordination. $T, X$ are independent with $T=R \mathbf{e}$ with $R \sim S^{1}(d, \mathcal{R})$ and $\mathbf{e}=$ $(1, \ldots, 1) \in \mathbb{R}^{n}$. We have $\mathbf{c}=\mathbf{0}$ in (2.9). Note $\left\langle\boldsymbol{\theta}_{1}, T(1)\right\rangle=R(1)\left\langle\boldsymbol{\theta}_{1}, \mathbf{e}\right\rangle$ and $\left(T(1) \diamond \Psi_{X}\right)\left(\boldsymbol{\theta}_{2}\right)=$ $R(1)\left(\mathbf{e} \diamond \Psi_{X}\left(\boldsymbol{\theta}_{2}\right)\right)$ in (3.8). Noting $\Re z \geq 0$ for $z:=-\mathrm{i}\left\langle\boldsymbol{\theta}_{1}, \mathbf{e}\right\rangle-\mathbf{e} \diamond \Psi_{X}\left(\boldsymbol{\theta}_{2}\right)$, we get from (3.8) that $\Psi_{(T, X \circ T)}(\boldsymbol{\theta})=-\Lambda_{R}(z)$, where $\Lambda_{R}(z):=d z+\int_{(0, \infty)}\left(1-e^{-z r}\right) \mathcal{R}(\mathrm{d} r)$. The RHS matches (3.1), and $T$ subordinates $X$ in the semi-strong sense.

Multivariate subordination. Let $T, X_{1}, X_{2}, \ldots, X_{n}$ be independent. Particularly, $\Sigma$ is a diagonal matrix and $\mathcal{X}=\sum_{k=1}^{n} \mathcal{X}_{\{k\}}$. If $\mathbf{t}=\left(t_{1}, \ldots, t_{n}\right) \in[0, \infty)^{n}, J_{(m)}:=\{(m), \ldots,(n)\}, 1 \leq m \leq n$, then (2.8) becomes $\mathbf{t} \diamond \mathcal{X}=\sum_{k=1}^{n} t_{k} \mathcal{X}_{\{k\}}$ as

$$
\sum_{m=1}^{n} \Delta t_{(m)}\left\{\sum_{k=1}^{n} \mathcal{X}_{\{k\}}\right\}_{J_{(m)}}=\sum_{k=1}^{n}\left\{\sum_{m=1}^{n} \Delta t_{(m)} \mathbf{1}_{J_{(m)}}(k)\right\} \mathcal{X}_{\{k\}}
$$


Note $\mathbf{c}=\mathbf{0}$ in (2.9) because for $\varnothing \neq J \subseteq\{1, \ldots, n\}, 1 \leq k \leq n$,

$$
\int_{\mathbb{D} C} \mathbf{x} \boldsymbol{\pi}_{J} \mathbf{1}_{\mathbb{D}}\left(\mathbf{x} \boldsymbol{\pi}_{J}\right) \mathcal{X}_{\{k\}}(\mathrm{d} \mathbf{x})=\mathbf{1}_{J}(k) \mathbf{e}_{k} \int_{\mathbb{D} C} x \mathbf{1}_{\mathbb{D}}(x) \mathcal{X}_{\{k\}}(\mathrm{d} x)=\mathbf{0} .
$$

Recalling the diagonal form of $\Sigma$ yields $\left\langle\boldsymbol{\theta}_{2}\left(\boldsymbol{\theta}_{2} \diamond \Sigma\right), \mathbf{t}\right\rangle=\left\|\boldsymbol{\theta}_{2}\right\|_{\mathbf{t} \diamond \Sigma}^{2}$ for $\mathbf{t} \in[0, \infty)^{n}, \boldsymbol{\theta}_{2} \in \mathbb{R}^{n}$. Also, $\Re \mathbf{z} \in[0, \infty)^{n}$, for $\boldsymbol{\theta}_{1}, \boldsymbol{\theta}_{2} \in \mathbb{R}^{n}$ and

$$
\begin{aligned}
\mathbf{z}:= & \frac{1}{2} \boldsymbol{\theta}_{2}\left(\boldsymbol{\theta}_{2} \diamond \Sigma\right)-\mathrm{i}\left(\boldsymbol{\theta}_{1}+\boldsymbol{\theta}_{2} \diamond \boldsymbol{\mu}\right) \\
& -\sum_{k=1}^{n} \mathbf{e}_{k} \int_{\mathbb{R}_{*}^{n}}\left(e^{\mathrm{i}\left\langle\boldsymbol{\theta}_{2}, \mathbf{x}\right\rangle}-1-\mathrm{i}\left\langle\boldsymbol{\theta}_{2}, \mathbf{x}\right\rangle \mathbf{1}_{\mathbb{D}}(\mathbf{x})\right) \mathcal{X}_{\{k\}}(\mathrm{d} \mathbf{x}) .
\end{aligned}
$$

By (3.8), note $\Psi_{(T, X \circ T)}(\boldsymbol{\theta})=-\Lambda_{T}(\mathbf{z})$, where $\Lambda_{T}(\mathbf{z}):=\langle\mathbf{z}, \mathbf{d}\rangle+\int_{[0, \infty)_{*}^{n}}\left(1-e^{-\langle\mathbf{z}, \mathbf{t}\rangle}\right) \mathcal{T}(\mathrm{d} \mathbf{t})$. As RHS matches (3.1), $T$ subordinates $X$ in the semi-strong sense.

Remark 3.2. Suppose $Y \stackrel{D}{=} B \odot T \sim W V \boldsymbol{\alpha} G^{n}(a, b, \boldsymbol{\alpha}, \boldsymbol{\mu}, \Sigma)$ in (2.19). If $\Sigma$ is of diagonal form, then $B$ is a Brownian motion with independent increments. Assume $B$ and $T$ are independent. Proposition 3.3 states that $Y \stackrel{D}{=} B \odot T \stackrel{D}{=} B \circ T \sim V \boldsymbol{\alpha} G^{n}(a, b, \boldsymbol{\alpha}, \boldsymbol{\mu}, \Sigma)$ in (2.18). Within the general class of $n$-dimensional Lévy processes, the $W V \boldsymbol{\alpha} G$-class is thus a proper extension of the strong $V \boldsymbol{\alpha} G$-class.

Monotone case. If its standard assumptions are violated, then traditional subordination may fail to create Lévy processes. Curiously, weak subordination overcomes this problem in the monotone case.

Proposition 3.4. Suppose $T, X$ be independent while $Z \stackrel{D}{=}(T, X \odot T)$ in the weak sense. If $T$ has monotone components $T_{1} \leq \cdots \leq T_{n}$, then $Z(t) \stackrel{D}{=}(T(t), X(T(t)))$ for all fixed $t \geq 0$.

Proof. Set $[0, \infty)_{\leq}^{n}:=\left\{\mathbf{t}=\left(t_{1}, \ldots, t_{n}\right) \in[0, \infty)^{n}: t_{1} \leq \ldots \leq t_{n}\right\}$. For $1 \leq k \leq n$, let $\Sigma_{k}=$ $\left(\Sigma_{k, i j}\right) \in \mathbb{R}^{n \times n}$ be defined by $\Sigma_{k, i j}:=\Sigma_{i j} \mathbf{1}\{i \wedge j \geq k\}$ for $1 \leq i, j \leq n$. For $\mathbf{t}=\left(t_{1}, \ldots, t_{n}\right) \in$ $[0, \infty)_{<}^{n}, 1 \leq k \leq n$, let $\Delta t_{k}:=t_{k}-t_{k-1}, t_{0}:=0$. The quantities in (2.7)-(2.9) are $\mathbf{t} \diamond \Sigma=$ $\sum_{k=1}^{n} \Delta t_{k} \Sigma_{k}, \mathbf{t} \diamond \mathcal{X}=\sum_{k=1}^{n} \Delta t_{k} \mathcal{X}_{\{k, \ldots, n\}}$ and $\mathbf{c}=\sum_{k=2}^{n} \Delta t_{k} \int_{\mathbb{D} C} \mathbf{x} \boldsymbol{\pi}_{\{k, \ldots, n\}} \mathbf{1}_{\mathbb{D}}\left(\mathbf{x} \boldsymbol{\pi}_{\{k, \ldots, n\}}\right) \mathcal{X}(\mathrm{d} \mathbf{x})$.

Introduce linear bijections $A, D: \mathbb{R}^{n} \rightarrow \mathbb{R}^{n}$ by setting

$$
\begin{aligned}
& \mathbf{x} A:=\left(x_{1}, x_{1}+x_{2}, x_{1}+x_{2}+x_{3}, \ldots, x_{1}+x_{2}+\cdots+x_{n}\right) \\
& \mathbf{x} D:=\left(x_{1}, x_{2}-x_{1}, x_{3}-x_{2}, \ldots, x_{n}-x_{n-1}\right), \quad \mathbf{x}=\left(x_{1}, \ldots, x_{n}\right) \in \mathbb{R}^{n}
\end{aligned}
$$

As we assumed $T_{1} \leq \cdots \leq T_{n}, T D \sim S^{n}\left(\mathbf{d} D, \mathcal{T} \circ D^{-1}\right)$ is a subordinator (see [21], his Theorem 24.11). 
Let $\boldsymbol{\theta}=\left(\boldsymbol{\theta}_{1}, \boldsymbol{\theta}_{2}\right), \boldsymbol{\theta}_{1}, \boldsymbol{\theta}_{2} \in \mathbb{R}^{n}$. Observe that $\Re \mathbf{z} \in[0, \infty)^{n}$, where

$$
\begin{aligned}
\mathbf{z}:= & -\mathrm{i} \boldsymbol{\theta}_{1} A^{\prime}-\mathrm{i}\left(\boldsymbol{\theta}_{2} \diamond \boldsymbol{\mu}\right) A^{\prime}+\frac{1}{2} \sum_{k=1}^{n}\left\|\boldsymbol{\theta}_{2}\right\|_{\Sigma_{k}}^{2} \mathbf{e}_{k} \\
& -\sum_{k=1}^{n} \int_{\mathbb{R}_{*}^{n}}\left(e^{\mathrm{i}\left\langle\boldsymbol{\theta}_{2}, \mathbf{x}\right\rangle}-1-\mathrm{i}\left\langle\boldsymbol{\theta}_{2}, \mathbf{x}\right\rangle \mathbf{1}_{\mathbb{D}}(\mathbf{x})\right) \mathcal{X}_{\{k, \ldots, n\}}(\mathrm{d} \mathbf{x}) \mathbf{e}_{k} \\
& -\mathrm{i} \sum_{k=2}^{n} \int_{\mathbb{D} C}\left\langle\boldsymbol{\theta}_{2}, \mathbf{x} \boldsymbol{\pi}_{\{k, \ldots, n\}}\right\rangle \mathbf{1}_{\mathbb{D}}\left(\mathbf{x} \boldsymbol{\pi}_{\{k, \ldots, n\}}\right) \mathcal{X}(\mathrm{d} \mathbf{x}) \mathbf{e}_{k} .
\end{aligned}
$$

As $A=D^{-1}$, note $\mathrm{i}\left\langle\boldsymbol{\theta}_{1}, \mathbf{t}\right\rangle+\mathbf{t} \diamond \Psi_{X}\left(\boldsymbol{\theta}_{2}\right)=-\langle\mathbf{z}, \mathbf{t} D\rangle$. Then using the assumption that $T$ and $X$ are independent, and the facts $\mathbf{d} \in[0, \infty)_{\leq}^{n}$ and $\mathcal{T}\left([0, \infty)_{*}^{n} \backslash[0, \infty)_{\leq}^{n}\right)=0$, it follows that $\Phi_{(T(t), X \circ T(t))}(\boldsymbol{\theta})=\exp \left\{-t \Lambda_{T D}(\mathbf{z})\right\}$ for $t \geq 0$, where $\Lambda_{T D}(\mathbf{z}):=\langle\mathbf{z}, \mathbf{d} D\rangle+\int_{[0, \infty)_{*}^{n}}(1-$ $\left.e^{-\langle\mathbf{z}, \mathbf{t} D\rangle}\right) \mathcal{T}(\mathrm{d} \mathbf{t})$ matches (3.1).

Remark 3.3. Let $B, B^{*}, I$ be the processes specified in Remark 2.2 so that $Z:=((I, 2 I)$, $(B, B) \odot(I, 2 I)) \stackrel{D}{=}\left(I, 2 I, B, B+B^{*}\right)$. The deterministic subordinator $(I, 2 I)$ satisfies $I \leq 2 I$. Proposition 3.4 matches $Z(t) \stackrel{D}{=}(t, 2 t, B(t), B(2 t))$ for all fixed $t \geq 0$.

Remark 3.4. Suppose $B$ is a standard Brownian motion and $N$ is a Poisson process with unit rate, independent of $B$. Note $\mathbb{E}[B(t) B(N(t))]=\mathbb{E}[t \wedge N(t)]=t\left(1-e^{-t}\right)$ for $0 \leq t \leq 1$, which is a nonlinear function in $t$. As a result, $(B, B) \circ(I, N)$ cannot be a Lévy process, and there is no Lévy process matching $(B, B) \circ(I, N)$ in law in all fixed time points $t \geq 0$. Neither $I \leq N$ nor $N \leq I$ holds for the subordinator $(I, N)$. It is verified from $(2.12)-(2.14)$ that $((I, N),(B, B) \odot$ $(I, N)) \stackrel{D}{=}\left((I, N),\left(B^{*}, B \circ N\right)\right)$ in the semi-strong sense, where $B^{*} \stackrel{D}{=} B$ is independent of $B, N$.

Ray-subordination. Recall $\mathbf{e}=(1, \ldots, 1) \in \mathbb{R}^{n}$, and let $(\mathbf{e}, \mathbf{e})=(1, \ldots, 1) \in \mathbb{R}^{2 n}$. If $\boldsymbol{\alpha} \in$ $[0, \infty)^{n}$ is a deterministic vector and $R$ is a univariate subordinator, then $T:=R \boldsymbol{\alpha}$ defines an $n$-dimensional subordinator travelling along the deterministic ray $\{r \alpha: r \geq 0\}$. We refer to this kind of subordination as ray-subordination. A special case is strong univariate subordination where the corresponding ray is given by $\{r \mathbf{e}: r \geq 0\}$.

Curiously, it is possible to perceive weak subordination along deterministic rays as univariate subordination of augmented processes.

Proposition 3.5. Let $\boldsymbol{\alpha} \in[0, \infty)^{n}$ be a deterministic vector and $R$ a univariate subordinator. If $Y$ is a Lévy process with characteristic exponent $\Psi_{Y}=\boldsymbol{\alpha} \diamond \Psi_{X}$, as in (2.11), but with $\mathbf{t}$ replaced by $\boldsymbol{\alpha}$, then we have $(R \boldsymbol{\alpha}, X \odot(R \boldsymbol{\alpha})) \stackrel{D}{=}(I \boldsymbol{\alpha}, Y) \odot(R(\mathbf{e}, \mathbf{e}))$.

If, in addition, $R$ and $Y$ are independent, then $(R \boldsymbol{\alpha}, X \odot(R \boldsymbol{\alpha})) \stackrel{D}{=}(I \boldsymbol{\alpha}, Y) \circ(R(\mathbf{e}, \mathbf{e}))$.

Proof. Let $\boldsymbol{\theta}=\left(\boldsymbol{\theta}_{1}, \boldsymbol{\theta}_{2}\right), \boldsymbol{\theta}_{1}, \boldsymbol{\theta}_{2} \in \mathbb{R}^{n}$. Suppose $R \sim S^{1}(d, \mathcal{R})$ and $\boldsymbol{\alpha}=\left(\alpha_{1}, \ldots, \alpha_{n}\right) \in[0, \infty)^{n}$. Without loss of generality, assume $\alpha_{1} \leq \cdots \leq \alpha_{n}$. Denote the augmented process by $W:=$ 
$(I \boldsymbol{\alpha}, Y)$. Proposition 2.1 states that $W(r)=(r \boldsymbol{\alpha}, Y(r)) \stackrel{D}{=}(r \boldsymbol{\alpha}, X(r \boldsymbol{\alpha}))$ for $r \geq 0$, thus proving the identity $I_{1}(\boldsymbol{\theta})=I_{2}(\boldsymbol{\theta})$, where $I_{1}(\boldsymbol{\theta}):=\int_{(0, \infty)}\left(\Phi_{(r \boldsymbol{\alpha}, X(r \boldsymbol{\alpha}))}(\boldsymbol{\theta})-1\right) \mathcal{R}(\mathrm{d} r)$ and $I_{2}(\boldsymbol{\theta}):=$ $\int_{(0, \infty)}\left(\Phi_{W(r(\mathbf{e}, \mathbf{e}))}(\boldsymbol{\theta})-1\right) \mathcal{R}(\mathrm{d} r)$.

Note $R \boldsymbol{\alpha} \sim S^{n}\left(d \boldsymbol{\alpha}, \mathcal{R} \circ(I \boldsymbol{\alpha})^{-1}\right)$. Proposition 3.1 and the transformation theorem tells us that $\Psi_{(R \boldsymbol{\alpha}, X \odot(R \boldsymbol{\alpha}))}(\boldsymbol{\theta})=\mathrm{i} d\left\langle\boldsymbol{\alpha}, \boldsymbol{\theta}_{1}\right\rangle+d \Psi_{Y}\left(\boldsymbol{\theta}_{2}\right)+I_{1}(\boldsymbol{\theta})$, also recalling $\boldsymbol{\alpha} \diamond \Psi_{X}\left(\boldsymbol{\theta}_{2}\right)=\Psi_{Y}\left(\boldsymbol{\theta}_{2}\right)$. Next, observe that $R(\mathbf{e}, \mathbf{e}) \sim S^{2 n}\left(d(\mathbf{e}, \mathbf{e}), \mathcal{R} \circ(I(\mathbf{e}, \mathbf{e}))^{-1}\right)$ and $\Psi_{W \odot(R(\mathbf{e}, \mathbf{e}))}(\boldsymbol{\theta})=\Psi_{(R(\mathbf{e}, \mathbf{e}), W \odot(R(\mathbf{e}, \mathbf{e})))}(\mathbf{0}$, $\boldsymbol{\theta})$. By Proposition 3.1 and the transformation theorem, the RHS evaluates to $d(\mathbf{e}, \mathbf{e}) \diamond \Psi_{W}(\boldsymbol{\theta})+$ $I_{2}(\boldsymbol{\theta})=\mathrm{i} d\left\langle\boldsymbol{\alpha}, \boldsymbol{\theta}_{1}\right\rangle+d \Psi_{Y}\left(\boldsymbol{\theta}_{2}\right)+I_{2}(\boldsymbol{\theta})$ by Proposition 2.1 .

The last statement in Proposition 3.5 follows from Proposition 3.3.

Remark 3.5. Let $B, B^{*}, N$ be independent processes, where $B \stackrel{D}{=} B^{*}$ are standard Brownian motions, and $N$ is a Poisson process with unit rate. By Proposition 3.5, it follows from independence that $((I, 2 I),(B, B) \odot(I, 2 I)) \stackrel{D}{=}\left(I, 2 I, B, B+B^{*}\right) \circ(I, I, I, I)$ and $((N, 2 N),(B, B) \odot$ $(N, 2 N)) \stackrel{D}{=}\left(I, 2 I, B, B+B^{*}\right) \circ(N, N, N, N)$. Thus, we can represent these processes using strong subordination with the univariate subordinators $I$ and $N$, respectively.

Moments. We give formulae for expected values and covariances.

Proposition 3.6. If $X$ and $T$ be as in Definition 2.1, then, for $t>0$,

$$
\begin{aligned}
\mathbb{E}[T(t)] / t & =\mathbf{d}+\int_{[0, \infty)_{*}^{n}} \mathbf{t} \mathcal{T}(\mathrm{d} \mathbf{t}), \quad \operatorname{Cov}(T(t)) / t=\int_{[0, \infty)_{*}^{n}} \mathbf{t}^{\prime} \mathbf{T}(\mathrm{d} \mathbf{t}), \\
\mathbb{E}[X \odot T(t)] / t & =\mathbf{d} \diamond \boldsymbol{\mu}+\int_{\mathbb{D} C} \mathbf{x}(\mathbf{d} \diamond \mathcal{X})(\mathrm{d} \mathbf{x})+\int_{[0, \infty)_{*}^{n}} \mathbb{E}[X(\mathbf{t})] \mathcal{T}(\mathrm{d} \mathbf{t}), \\
\operatorname{Cov}(X \odot T(t)) / t & =\mathbf{d} \diamond \Sigma+\int_{\mathbb{R}_{*}^{n}} \mathbf{x}^{\prime} \mathbf{x}(\mathbf{d} \diamond \mathcal{X})(\mathrm{d} \mathbf{x})+\int_{[0, \infty)_{*}^{n}} \mathbb{E}\left[X^{\prime}(\mathbf{t}) X(\mathbf{t})\right] \mathcal{T}(\mathrm{d} \mathbf{t}), \\
\operatorname{Cov}(X \odot T(t), T(t)) / t & =\int_{[0, \infty)_{*}^{n}} \mathbb{E}\left[X^{\prime}(\mathbf{t})\right] \mathbf{t} \mathcal{T}(\mathrm{d} \mathbf{t}),
\end{aligned}
$$

provided the participating integrals are finite.

Proof. Given the characteristics of $Z \stackrel{D}{=}(T, X \odot T) \sim L^{2 n}(\mathbf{m}, \Theta, \mathcal{Z})$ in (2.12)-(2.15), these follow from the general formulae for moments of Lévy processes (see [21], his Example 25.12).

Remark 3.6. Let Brownian motion $B \sim B M^{n}(\boldsymbol{\mu}, \Sigma)$ be the weak subordinate and $T \sim S^{n}(\mathbf{d}, \mathcal{T})$ be the subordinator. By Proposition 3.6, for $1 \leq k \leq n$,

$$
\begin{aligned}
\mathbb{E}\left[(B \odot T)_{k}(1)\right] & =\mu_{k} \mathbb{E}\left[T_{k}(1)\right], \\
\operatorname{Var}\left((B \odot T)_{k}(1)\right) & =\Sigma_{k k} \mathbb{E}\left[T_{k}(1)\right]+\mu_{k}^{2} \operatorname{Var}\left(T_{k}(1)\right) .
\end{aligned}
$$


Assume $1 \leq k \neq l \leq n, u>0$, and set

$$
\tau_{k, l}(u):=\mathcal{T}\left(\left\{\mathbf{t}=\left(t_{1}, \ldots, t_{n}\right) \in[0, \infty)_{*}^{n}: t_{k} \wedge t_{l}>u\right\}\right) .
$$

Recall $s \wedge t=\int_{(0, \infty)} \mathbf{1}_{(u, \infty)}(s) \mathbf{1}_{(u, \infty)}(t) \mathrm{d} u, s, t \geq 0$, and $\int_{[0, \infty)_{*}^{n}} t_{k} \wedge t_{l} \mathcal{T}(\mathrm{d} \mathbf{t})=\int_{(0, \infty)} \tau_{k, l}(u) \mathrm{d} u$. Proposition 3.6 states that

$$
\begin{aligned}
\operatorname{Cov}\left((B \odot T)_{k}(1),(B \odot T)_{l}(1)\right)= & \mu_{k} \mu_{l} \operatorname{Cov}\left(T_{k}(1), T_{l}(1)\right)+\Sigma_{k l}\left(d_{k} \wedge d_{l}\right) \\
& +\Sigma_{k l} \int_{(0, \infty)} \tau_{k, l}(u) \mathrm{d} u .
\end{aligned}
$$

Remark 3.7. Let $B \odot T \sim W V \boldsymbol{\alpha} G^{n}(a, b, \boldsymbol{\alpha}, \boldsymbol{\mu}, \Sigma)$ be as in (2.19). As the components of $T=$ $\left(T_{1}, \ldots, T_{n}\right)$ are standard gamma subordinators $T_{k} \sim \Gamma_{S}\left(b / \alpha_{k}\right), 1 \leq k \leq n$, the first and second moments of an $\alpha G$-subordinator are determined as follows (see [23]),

$$
\mathbb{E}\left[T_{k}(1)\right]=1, \quad \operatorname{Var}\left(T_{k}(1)\right)=\alpha_{k} / b, \quad 1 \leq k \leq n,
$$

and

$$
\operatorname{Cov}\left(T_{k}(1), T_{l}(1)\right)=\alpha_{k} \alpha_{l} \operatorname{Var}\left(T_{0}(1)\right)=\alpha_{k} \alpha_{l} a / b^{2}, \quad 1 \leq k \neq l \leq n .
$$

It follows from Remark 3.6 that, for $1 \leq k \leq n$,

$$
\mathbb{E}\left[(B \odot T)_{k}(1)\right]=\mu_{k}, \quad \operatorname{Var}\left((B \odot T)_{k}(1)\right)=\left(b \Sigma_{k k}+\mu_{k}^{2} \alpha_{k}\right) / b,
$$

and these formulae match, not surprisingly, those of univariate $V G^{1}$-processes in [16] because of (3.7). If $1 \leq k \neq l \leq n$, observe $\int_{(0, \infty)} \tau_{k, l ; a, b, \boldsymbol{\alpha}}(u) \mathrm{d} u=\left(\alpha_{k} \wedge \alpha_{l}\right) \mathbb{E}\left[T_{0}(1)\right]=\left(\alpha_{k} \wedge \alpha_{l}\right) a / b$, with covariance given by

$$
\begin{aligned}
& \operatorname{Cov}\left((B \odot T)_{k}(1),(B \odot T)_{l}(1)\right) \\
& \quad=\Sigma_{k l}\left(\alpha_{k} \wedge \alpha_{l}\right) \mathbb{E}\left[T_{0}(1)\right]+\mu_{k} \mu_{l} \operatorname{Cov}\left(T_{k}(1), T_{l}(1)\right) \\
& \quad=\left(a b\left(\alpha_{k} \wedge \alpha_{l}\right) \Sigma_{k l}+a \alpha_{k} \alpha_{l} \mu_{k} \mu_{l}\right) / b^{2}
\end{aligned}
$$

These moments for the $W V \boldsymbol{\alpha} G^{n}$-process have also been derived in [18] as well as higher moments.

For traditional subordination, (3.9) reduces to $\alpha_{k} \alpha_{l} \mu_{k} \mu_{l} / b^{2}$ (see [23], her Section 4) as $\Sigma$ is diagonal, which was noted as a disadvantage in [12,14]. In contrast, $B \odot T$ has an additional correlation term which includes the correlation of the Brownian motion.

Superposition. If a process $X$ is weakly subordinated by a superposition of several independent subordinators, then its law equals the sum of independent Lévy processes.

Proposition 3.7. Let $X$ be an $n$-dimensional Lévy process. Let $\mathbf{d} \in[0, \infty)^{n}$ be a deterministic vector. If $T_{1}, \ldots, T_{m}$ are independent $n$-dimensional drift-less subordinators, then $T:=$ 
$I \mathbf{d}+\sum_{k=1}^{m} T_{k}$ is an n-dimensional subordinator with drift $\mathbf{d}$ and $(T, X \odot T) \stackrel{D}{=} \sum_{j=0}^{m} A_{j}$, where $A_{0}, A_{1}, \ldots, A_{m}$ are independent Lévy processes with $A_{0} \stackrel{D}{=}(I \mathbf{d}, X \odot I \mathbf{d}), A_{k} \stackrel{D}{=}\left(T_{k}, X \odot T_{k}\right)$, $1 \leq k \leq m$.

Proof. Assume that $T_{1}, \ldots, T_{m}, A_{0}, \ldots, A_{m}$ are independent processes, where $T_{k} \sim S^{n}\left(\mathbf{0}, \mathcal{T}_{k}\right)$, $A_{0} \stackrel{D}{=}(I \mathbf{d}, X \odot I \mathbf{d})$ and $A_{k} \stackrel{D}{=}\left(T_{k}, X \odot T_{k}\right), 1 \leq k \leq m$. In particular, note $T \sim S^{n}\left(\mathbf{d}, \sum_{k=1}^{m} \mathcal{T}_{k}\right)$, then by (3.1),

$$
\begin{aligned}
\Psi_{(T, X \odot T)}(\boldsymbol{\theta}) & =\mathrm{i}\left\langle\boldsymbol{\theta}_{1}, \mathbf{d}\right\rangle+\left(\mathbf{d} \diamond \Psi_{X}\right)\left(\boldsymbol{\theta}_{2}\right)+\int_{[0, \infty)_{*}^{n}}\left(\Phi_{(\mathbf{t}, X(\mathbf{t}))}(\boldsymbol{\theta})-1\right)\left(\sum_{k=1}^{m} \mathcal{T}_{k}\right)(\mathrm{d} \mathbf{t}) \\
& =\sum_{k=0}^{m} \Psi_{A_{k}}(\boldsymbol{\theta})=\Psi_{\sum_{k=0}^{m} A_{k}}(\boldsymbol{\theta}), \quad \boldsymbol{\theta}=\left(\boldsymbol{\theta}_{1}, \boldsymbol{\theta}_{2}\right), \boldsymbol{\theta}_{1}, \boldsymbol{\theta}_{2} \in \mathbb{R}^{n}
\end{aligned}
$$

as desired.

Remark 3.8. In the context of traditional subordination (see [7], their Proposition 4.1), Proposition 3.7 holds without assuming drift-less subordinators. This is more delicate when dealing with weak subordination. Let $B, B^{*}, W, W^{*}$ be independent standard univariate Brownian motions.

Remark 2.2 states that $(B, B) \odot(I, 2 I) \stackrel{D}{=}\left(B, B+B^{*}\right)$ and $(B, B) \odot(2 I, I) \stackrel{D}{=}\left(W+W^{*}, W\right)$. Proposition 3.3 states that $(B, B) \odot(3 I, 3 I) \stackrel{D}{=}(B, B) \circ(3 I, 3 I)$. Note $\left(B, B+B^{*}\right)+(W+$ $\left.W^{*}, W\right) \sim B M^{2}(\mathbf{0},[(3,2),(2,3)])$ and $(B, B) \circ(3 I, 3 I) \sim B M^{2}(\mathbf{0},[(3,3),(3,3)])$. There are no independent processes $Y_{1}, Y_{2}$ such that, simultaneously, $Y_{1} \stackrel{D}{=}(B, B) \odot(I, 2 I), Y_{2} \stackrel{D}{=}(B, B) \odot$ $(2 I, I))$ and $Y_{1}+Y_{2} \stackrel{D}{=}(B, B) \odot(3 I, 3 I)$.

Remark 3.9. Let $B \odot T \sim W V \boldsymbol{\alpha} G^{n}(a, b, \boldsymbol{\alpha}, \boldsymbol{\mu}, \Sigma)$ in (2.19). We derive a joint representation of $(T, B \odot T)$ in terms of a superposition of gamma processes and variance-gamma processes. Let $B, B^{(1)}, \ldots, B^{(n)}, W^{(\alpha)}, G_{0}, \ldots, G_{n}$ be independent, where $B^{(1)}, \ldots, B^{(n)}$ are copies of $B \sim$ $B M^{n}(\boldsymbol{\mu}, \Sigma), G_{0}, \ldots, G_{n}$ are as in (2.16) and $W^{(\boldsymbol{\alpha})} \sim B M^{n}(\boldsymbol{\alpha} \diamond \boldsymbol{\mu}, \boldsymbol{\alpha} \diamond \Sigma)$ is a Brownian motion.

Next, standardise $b G_{0} / a \sim \Gamma_{S}(a)$ and $\left(b /\left(b-a \alpha_{k}\right)\right) G_{k} \sim \Gamma_{S}\left(\beta_{k}\right)$ to see that $V_{0}:=W^{(\boldsymbol{\alpha})}$ 。 $\left(G_{0} \mathbf{e}\right) \sim V G^{n}(a,(a / b)(\boldsymbol{\alpha} \diamond \boldsymbol{\mu}, \boldsymbol{\alpha} \diamond \Sigma))$ and $V_{k}:=B_{k}^{(k)} \circ G_{k} \sim V G^{1}\left(\beta_{k},\left(\left(b-a \alpha_{k}\right) / b\right)\left(\mu_{k}, \Sigma_{k k}\right)\right)$, $1 \leq k \leq n$. Note $V_{0}, \ldots, V_{n}$ are independent.

Plainly, $T$ in (2.16) is the superposition of independent univariate gamma processes travelling along deterministic rays generated by $\boldsymbol{\alpha}, \mathbf{e}_{1}, \ldots, \mathbf{e}_{n} \in[0, \infty)_{*}^{n}$. Combining Propositions 3.7 and 3.5 yields, for $Z \stackrel{D}{=}(T, B \odot T)$,

$$
\begin{aligned}
& Z \stackrel{D}{=}\left(G_{0} \boldsymbol{\alpha}, B \odot\left(G_{0} \boldsymbol{\alpha}\right)\right)+\sum_{k=1}^{n}\left(G_{k} \mathbf{e}_{k}, B^{(k)} \odot\left(G_{k} \mathbf{e}_{k}\right)\right) \\
& \quad \stackrel{D}{=}\left(I \boldsymbol{\alpha}, W^{(\boldsymbol{\alpha})}\right) \circ\left(G_{0}(\mathbf{e}, \mathbf{e})\right)+\sum_{k=1}^{n}\left(G_{k} \mathbf{e}_{k},\left(B_{k}^{(k)} \circ G_{k}\right) \mathbf{e}_{k}\right)=\left(G_{0} \boldsymbol{\alpha}, V_{0}\right)+\sum_{k=1}^{n}\left(G_{k} \mathbf{e}_{k}, V_{k} \mathbf{e}_{k}\right) .
\end{aligned}
$$


Our $W V \boldsymbol{\alpha} G$-process satisfies $Y \stackrel{D}{=} B \odot T \stackrel{D}{=} V_{0}+\sum_{k=1}^{n} V_{k} \mathbf{e}_{k}$ as the superposition of independent $V G^{n}$-processes (for the strong formulation, see [7], their Remark 2.17).

Subordinators with independent components. If a drift-less subordinator has independent components, then so does any associated weakly subordinated process.

Proposition 3.8. Let $X$ and $T$ be as in Definition 2.1, with drift-less $T$. If the components of $T$ are independent, then so are those of $X \odot T$.

Proof. If $T=\left(T_{1}, \ldots, T_{n}\right) \sim S^{n}(\mathbf{0}, \mathcal{T})$ has independent components $T_{1} \sim S^{1}\left(0, \mathcal{T}_{1}\right), \ldots, T_{n} \sim$ $S^{1}\left(0, \mathcal{T}_{n}\right)$, then $\mathcal{T}=\sum_{k=1}^{n} \delta_{0}^{\otimes(k-1)} \otimes \mathcal{T}_{k} \otimes \delta_{0}^{\otimes(n-k)}$. In (3.4)-(3.5), note $\mathbf{d} \diamond \Sigma=0$ and $\mathcal{Z}_{2}=$ $\sum_{k=1}^{n} \boldsymbol{\delta}_{0}^{\otimes(k-1)} \otimes \mathcal{Y}_{k} \otimes \delta_{0}^{\otimes(n-k)}$, where $\mathcal{Z}_{2}, \mathcal{Y}_{1}, \ldots, \mathcal{Y}_{n}$ are the Lévy measures corresponding to $X \odot T, X_{1} \odot T_{1}, \ldots, X_{n} \odot T_{n}$, as required.

Remark 3.10. If $B, B^{*}, N, N^{*}$ are independent processes, such that $B, B^{*}$ are univariate standard Brownian motions and $N, N^{*}$ are Poisson processes with unit rate, then it is straightforwardly verified from (3.3)-(3.5) and Proposition 3.3 that $(B, B) \odot\left(N, N^{*}\right) \stackrel{D}{=}\left(B \circ N, B^{*} \circ N^{*}\right)$ decomposes into a Lévy process with independent compound Poisson components.

We have previously listed sufficient conditions for strong subordination [3,21] to stay in the class of Lévy processes. Next, we show that these conditions are necessary in some cases (see Section 5.3 for a proof).

Proposition 3.9. Let $T=\left(T_{1}, T_{2}\right)$ and $X=\left(X_{1}, X_{2}\right)$ be independent bivariate Lévy processes, where $T$ is a subordinator. Suppose neither $T_{1} \equiv 0$ nor $T_{2} \equiv 0$. If $X \circ T$ is also a Lévy process, then $T_{1}=T_{2}$ must be indistinguishable, provided one of the following holds in addition:

(i) $X \stackrel{D}{=}-X$ is symmetric, and $X_{1}, X_{2}$ are dependent;

(ii) $T$ is deterministic, and $X_{1}, X_{2}$ are dependent;

(iii) $T$ admits a finite first moment, and $X$ admits a finite second moment with correlated components $X_{1}, X_{2}$.

In Proposition 3.4, we stated monotonicity as a sufficient condition ensuring that the weakly subordinated process matches the marginal distributions of the strongly subordinated one. Next, we show that for this purpose, monotonicity is needed in some cases (see Section 5.3 for a proof).

Proposition 3.10. If $T=\left(T_{1}, T_{2}\right), X=\left(X_{1}, X_{2}\right)$ and $Y=\left(Y_{1}, Y_{2}\right)$ are bivariate Lévy processes, where $T, X$ are independent and $T$ is a subordinator, while $X$ has dependent components $X_{1}, X_{2}$, then there is at least one $t \in(0, \infty)$ violating $X(T(t)) \stackrel{D}{=} Y(t)$, provided one of following holds in addition:

(i) both $T, X$ admit finite second moments, while $X$ has correlated components and $T$ has non-monotonic components; 
(ii) $Y \stackrel{D}{=} X \odot T, X$ is symmetric, while $T_{1}, T_{2}$ are independent, drift-less and nontrivial subordinators.

Remark 3.11. In Proposition 3.10(ii), the subordinator has independent and non-deterministic components, and so is non-monotonic, that is, neither $T_{1}-T_{2}$ nor $T_{2}-T_{1}$ is a subordinator.

It would be interesting to see whether or not the conditions in Propositions 3.9-3.10 could be further weakened. We speculate that this extension is possible based on Dynkin-type formulae and fluctuation theory for Lévy processes. We have to leave this as an interesting avenue of future research.

\section{Variance generalised gamma convolutions}

In this section, the weak subordinate is Brownian motion $B \sim B M^{n}(\boldsymbol{\mu}, \Sigma)$, and $T \sim S^{n}(\mathbf{d}, \mathcal{T})$ is the subordinator. Since the Lévy measure of $B$ is 0 , we get simplifications in (2.8)-(2.15) and (3.3)-(3.5). The weakly subordinated process is denoted by $Y \stackrel{D}{=} B \odot T \sim L^{n}\left(\mathbf{m}_{2}, \mathbf{d} \diamond \Sigma, \mathcal{Y}\right)$.

Thorin [24,25] characterised the class of generalised gamma convolutions $(G G C)$ as the subset of univariate Borel probability measures containing arbitrary finite convolutions of gamma distributions, while being closed under convergence in distribution (see the survey article [13] and the monograph [22]). Multivariate extensions of these results and examples have been investigated in $[2,6,19]$, and these are subclasses of the self-decomposable and, thus, infinitely divisible distributions. Our subordinators will be taken from this class.

Thorin subordinator. In our exposition, we follow [7]. Recall $\ln ^{-} x=-\mathbf{1}_{(0,1]}(x) \ln x, x>0$. A nonnegative Borel measure $\mathcal{U}$ on $[0, \infty)_{*}^{n}$ is called an $n$-dimensional Thorin measure, provided

$$
\int_{[0, \infty)_{*}^{n}}\left(1+\ln ^{-}\|\mathbf{u}\|\right) \wedge(1 /\|\mathbf{u}\|) \mathcal{U}(\mathrm{d} \mathbf{u})<\infty
$$

If $\mathbf{d} \in[0, \infty)^{n}$ and $\mathcal{U}$ is a Thorin measure, we call an $n$-dimensional subordinator $T$ a Thorin subordinator, in brief $T \sim G G C_{S}^{n}(\mathbf{d}, \mathcal{U})$, whenever, for all $t \geq 0, \lambda \in[0, \infty)^{n}$, it has Laplace exponent

$$
-\ln \mathbb{E} \exp \{-\langle\lambda, T(t)\rangle\}=t\langle\mathbf{d}, \lambda\rangle+t \int_{[0, \infty)_{*}^{n}} \ln \left\{\left(\|\mathbf{u}\|^{2}+\langle\lambda, \mathbf{u}\rangle\right) /\|\mathbf{u}\|^{2}\right\} \mathcal{U}(\mathrm{du})
$$

The distribution of a Thorin subordinator is uniquely determined by $\mathbf{d}$ and $\mathcal{U}$.

Let $\mathbb{S}_{+}:=\mathbb{S} \cap[0, \infty)_{*}^{n}$, where $\mathbb{S}:=\left\{\mathbf{s} \in \mathbb{R}^{n}:\|\mathbf{s}\|=1\right\}$ is the unit sphere. If $T \sim S^{n}(\mathbf{d}, \mathcal{T})$, the Lévy measure $\mathcal{T}$ is derived using a polar-decomposition of its Thorin measure. Specifically, if $A \in[0, \infty)_{*}^{n}$ is a Borel set, then we may write (see [7], their Lemma 4.1)

$$
\mathcal{U}(A)=(\mathcal{S} \otimes \mathcal{K}) \circ((\mathbf{s}, r) \mapsto r \mathbf{s})^{-1}(A)=\int_{\mathbb{S}_{+}} \int_{(0, \infty)} \mathcal{K}(\mathbf{s}, \mathrm{d} r) \mathbf{1}_{A}(r \mathbf{s}) \mathcal{S}(\mathrm{d} \mathbf{s})
$$


Here, $\mathcal{S}$ is a finite nonnegative Borel measure on $\mathbb{S}_{+}$and $\mathcal{K}$ is a Thorin kernel, that is a nonnegative Borel kernel with

$$
0<\int_{(0, \infty)}\left(1+\ln ^{-} r\right) \wedge(1 / r) \mathcal{K}(\mathbf{s}, \mathrm{d} r)<\infty, \quad \mathbf{s} \in \mathbb{S}_{+} .
$$

Recall $\mathcal{G}_{b}$ is the Lévy measure of a standard gamma subordinator with shape parameter $b$.

Lemma 4.1. If $T \sim G G C_{S}^{n}(\mathbf{d}, \mathcal{U})$, then $T \sim S^{n}(\mathbf{d}, \mathcal{T})$, where

$$
\mathcal{T}=\left\{\frac{\mathcal{U}(\mathrm{d} \mathbf{u})}{\|\mathbf{u}\|^{2}} \otimes \mathcal{G}_{\|\mathbf{u}\|^{2}}(\mathrm{~d} g)\right\} \circ((\mathbf{u}, g) \mapsto g \mathbf{u})^{-1} .
$$

Proof. If $T \sim G G C_{S}^{n}(\mathbf{d}, \mathcal{U})$, then $T \sim S^{n}(\mathbf{d}, \mathcal{T})$ in polar coordinates is (see [7], their Equations (2.17)-(2.18))

$$
\begin{aligned}
\mathcal{T}(A) & =\int_{\mathbb{S}^{+}} \int_{(0, \infty)} \mathbf{1}_{A}(r \mathbf{s}) k(\mathbf{s}, r) \frac{\mathrm{d} r}{r} \mathcal{S}(\mathrm{d} \mathbf{s}), \quad A \subseteq[0, \infty)_{*}^{n} \text { Borel }, \\
k(\mathbf{s}, r) & =\int_{(0, \infty)} e^{-r v} \mathcal{K}(\mathbf{s}, \mathrm{d} v), \quad r>0, \mathbf{s} \in \mathbb{S}_{+} .
\end{aligned}
$$

If $A \subseteq[0, \infty)^{n}$ is Borel, by using (4.3) and making the substitution $g=r /\|\mathbf{u}\|$, we get that

$$
\mathcal{T}(A)=\int_{[0, \infty)_{*}^{n}}\left\{\|\mathbf{u}\|^{2} \int_{(0, \infty)} \mathbf{1}_{A}(g \mathbf{u}) e^{-g\|\mathbf{u}\|^{2}} \frac{\mathrm{d} g}{g}\right\} \frac{\mathcal{U}(\mathrm{d} \mathbf{u})}{\|\mathbf{u}\|^{2}} .
$$

Here, the RHS matches the RHS of (4.2) when evaluated at $A$.

Remark 4.1. If $T \sim \boldsymbol{\alpha} G^{n}(a, b, \boldsymbol{\alpha})$ in (2.16), then $T$ is determined as the superposition of independent gamma subordinators $G_{0}, \ldots, G_{n}$, travelling along rays generated by $\boldsymbol{\alpha}, \mathbf{e}_{1}, \ldots, \mathbf{e}_{n}$, respectively. Recall $\beta_{k}:=\left(b-a \alpha_{k}\right) / \alpha_{k}, 1 \leq k \leq n$. If $\lambda \in[0, \infty)_{*}^{n}$, then

$$
\begin{aligned}
& -\ln \mathbb{E} \exp \{-\langle\lambda, T(t)\rangle\} \\
& =-\ln \mathbb{E}\left[\exp \left\{-G_{0}(t)\langle\lambda, \boldsymbol{\alpha}\rangle\right\}\right]-\sum_{k=1}^{n} \ln \mathbb{E}\left[\exp \left\{-G_{k}(t)\left\langle\lambda, \mathbf{e}_{k}\right\rangle\right\}\right] \\
& =\text { at } \ln \{(b+\langle\lambda, \boldsymbol{\alpha}\rangle) / b\}+\sum_{k=1}^{n} \beta_{k} t \ln \left\{\left(\left(b / \alpha_{k}\right)+\left\langle\lambda, \mathbf{e}_{k}\right\rangle\right) /\left(b / \alpha_{k}\right)\right\} .
\end{aligned}
$$

Here, we used independence and the Laplace exponent of the underlying gamma subordinators.

The RHS matches (4.1) for $\mathbf{d}=\mathbf{0}$ and $\mathcal{U}_{a, b, \boldsymbol{\alpha}}:=a \boldsymbol{\delta}_{b \boldsymbol{\alpha} /\|\boldsymbol{\alpha}\|^{2}}+\sum_{k=1}^{n} \beta_{k} \boldsymbol{\delta}_{b \mathbf{e}_{k} / \alpha_{k}}$. Therefore, $\mathcal{U}_{a, b, \boldsymbol{\alpha}}$ defines a finitely supported Thorin measure, and $T \sim G G C_{S}^{n}\left(\mathbf{0}, \mathcal{U}_{a, b, \boldsymbol{\alpha}}\right)$ is a drift-less Thorin subordinator.

Using $\mathcal{U}_{a, b, \boldsymbol{\alpha}}$ and (4.2), it is possible to give an alternative derivation of the Lévy measure $\mathcal{T}_{a, b, \alpha}$ in (2.17) (see [7] and [14], their Lemma 2.13 and their Theorem 1.1, respectively). 
Variance generalised gamma convolutions. For the parameters of this model, we assume an $n$-dimensional Thorin measure $\mathcal{U}, \boldsymbol{\mu} \in \mathbb{R}^{n}, \mathbf{d} \in[0, \infty)^{n}$ and a covariance matrix $\Sigma \in \mathbb{R}^{n \times n}$. Let $B \sim B M^{n}(\mu, \Sigma)$ be a Brownian motion. Let $T \sim G G C_{S}^{n}(\mathbf{d}, \mathcal{U})$. Given such $B$ and $T$, we call a Lévy process of the form $Y \stackrel{D}{=} B \odot T$ an $n$-dimensional variance generalised gamma convolution $\left(V G G^{n}\right)$ process with parameters $\mathbf{d}, \boldsymbol{\mu}, \Sigma, \mathcal{U}$. We write this as

$$
Y \sim V G G^{n}(\mathbf{d}, \boldsymbol{\mu}, \Sigma, \mathcal{U}):=B M^{n}(\boldsymbol{\mu}, \Sigma) \odot G G C_{S}^{n}(\mathbf{d}, \mathcal{U})
$$

Theorem 2.1 ensures the existence of $Y \sim V G G^{n}(\mathbf{d}, \boldsymbol{\mu}, \Sigma, \mathcal{U})$.

Characteristics. We derive formulae of the characteristic exponent and the Lévy measure, valid within the $V G G^{n}$-class. If $\varnothing \neq J \subseteq\{1, \ldots, n\}$, introduce $C_{J} \subseteq V_{J} \subseteq \mathbb{R}^{n}$, where $\mathbf{u}=$ $\left(u_{1}, \ldots, u_{n}\right) \in C_{J}$ and $\mathbf{y}=\left(y_{1}, \ldots, y_{n}\right) \in V_{J}$ if and only if $u_{j}>0$ for all $j \in J$ and $y_{j} \neq 0$ for all $j \in J$, respectively. If $\mathbf{u} \in C_{J}$, while $\Sigma$ is invertible, the restriction $(\mathbf{u} \diamond \Sigma)_{J}: \mathbb{R}^{n} \boldsymbol{\pi}_{J} \rightarrow \mathbb{R}^{n} \boldsymbol{\pi}_{J}$, $\mathbf{x} \mapsto \mathbf{x}(\mathbf{u} \diamond \Sigma)_{J}:=\mathbf{x}(\mathbf{u} \diamond \Sigma)$ is an invertible linear mapping, thus having inverse $(\mathbf{u} \diamond \Sigma)_{J}^{-1}$ and determinant $|\mathbf{u} \diamond \Sigma|_{J}$.

Theorem 4.1. If $Y \sim V G G^{n}(\mathbf{d}, \boldsymbol{\mu}, \Sigma, \mathcal{U})$, then $Y \sim L^{n}\left(\mathbf{m}_{2}, \mathbf{d} \diamond \Sigma, \mathcal{Y}\right)$, where $\mathbf{m}_{2}=\mathbf{d} \diamond \boldsymbol{\mu}+$ $\int_{\mathbb{D}_{*}} \mathbf{y} \mathcal{Y}(\mathrm{d} \mathbf{y}), \mathcal{V}$ is the quantity in (2.5) and

$$
\mathcal{Y}=\left\{\frac{\mathcal{U}(\mathrm{d} \mathbf{u})}{\|\mathbf{u}\|^{2}} \otimes \mathcal{V}_{\|\mathbf{u}\|^{2}, \mathbf{u} \diamond \mu, \mathbf{u} \diamond \Sigma}(\mathrm{d} \mathbf{y})\right\} \circ((\mathbf{u}, \mathbf{y}) \mapsto \mathbf{y})^{-1},
$$

and, for $\boldsymbol{\theta} \in \mathbb{R}^{n}$,

$$
\begin{aligned}
\Psi_{Y}(\boldsymbol{\theta})=\mathrm{i}\langle\mathbf{d} \diamond \boldsymbol{\mu}, \boldsymbol{\theta}\rangle-\frac{1}{2}\|\boldsymbol{\theta}\|_{\mathbf{d} \diamond \Sigma}^{2} \\
-\int_{[0, \infty)_{*}^{n}} \ln \left\{\left(\|\mathbf{u}\|^{2}-\mathrm{i}\langle\mathbf{u} \diamond \boldsymbol{\mu}, \boldsymbol{\theta}\rangle+\frac{1}{2}\|\boldsymbol{\theta}\|_{\mathbf{u} \diamond \Sigma}^{2}\right) /\|\mathbf{u}\|^{2}\right\} \mathcal{U}(\mathrm{d} \mathbf{u}) .
\end{aligned}
$$

If, in addition, $\Sigma$ is invertible, then $\mathcal{Y}=\sum_{\varnothing \neq J \subseteq\{1, \ldots, n\}} \mathcal{Y}_{J}, \mathcal{Y}_{J}\left(\mathbb{R}_{J} \backslash V_{J}\right)=0$, where $\mathcal{Y}_{J}$ is absolutely continuous with respect to $\mathrm{d} \mathbf{y} \circ \pi_{J}^{-1}$ having density $v_{J}(\mathbf{y})=\int_{C_{J}} v_{J}(\mathbf{u}, \mathbf{y}) \mathcal{U}(\mathrm{du})$, where $\mathbf{u} \in C_{J}, \mathbf{y} \in V_{J}, c_{J}:=2 /(2 \pi)^{\# J / 2}$, and

$$
\begin{aligned}
v_{J}(\mathbf{u}, \mathbf{y})= & c_{J} \mathfrak{K}_{\# J / 2}\left\{\left[\|\mathbf{y}\|_{(\mathbf{u} \diamond \Sigma)_{J}^{-1}}\left(2\|\mathbf{u}\|^{2}+\|\mathbf{u} \diamond \boldsymbol{\mu}\|_{(\mathbf{u} \diamond \Sigma)_{J}^{-1}}^{2}\right)^{1 / 2}\right\}\right. \\
& \times \exp \left\{\langle\mathbf{y}, \mathbf{u} \diamond \boldsymbol{\mu}\rangle_{(\mathbf{u} \diamond \Sigma)_{J}^{-1}}\right\} /\left\{|\mathbf{u} \diamond \Sigma|_{J}^{1 / 2}\|\mathbf{y}\|_{(\mathbf{u} \diamond \Sigma)_{J}^{-1}}^{n}\right\} .
\end{aligned}
$$

Proof. The formulae of the triplet $\left(\mathbf{m}_{2}, \mathbf{d} \diamond \Sigma, \mathcal{Y}\right)$ follow from Proposition 3.2. To see this, let $A \subseteq \mathbb{R}_{*}^{n}$ be a Borel set. Combining (2.5) with Proposition 2.1 yields $\int_{(0, \infty)} \mathbb{P}(B(g \mathbf{u}) \in$ $A) \mathcal{G}_{\|\mathbf{u}\|^{2}}(\mathrm{~d} g)=\mathcal{V}_{\|\mathbf{u}\|^{2}, \mathbf{u} \diamond \boldsymbol{\mu}, \mathbf{u} \diamond \Sigma}(A)$. In particular, we get from (3.5) and (4.2) that

$$
\mathcal{Y}(A)=\int_{[0, \infty)_{*}^{n}} \int_{(0, \infty)} \mathbb{P}(B(g \mathbf{u}) \in A) \mathcal{G}_{\|\mathbf{u}\|^{2}}(\mathrm{~d} g) \frac{\mathcal{U}(\mathrm{d} \mathbf{u})}{\|\mathbf{u}\|^{2}},
$$

where the RHS matches the RHS in (4.4) when evaluated at $A$. 
As $\mathbf{t} \mapsto \mathbb{E}\left[B(\mathbf{t}) \mathbf{1}_{\mathbb{D}_{*}}(B(\mathbf{t}))\right]$ is $\mathcal{T}$-integrable by (2.3) and (5.4), $\mathbf{y} \mapsto \mathbf{y} \mathbf{1}_{\mathbb{D}_{*}}(\mathbf{y})$ is $\mathcal{Y}(\mathrm{d} \mathbf{y})=$ $\mathbb{P}(B(\mathbf{t}) \in \mathrm{d} \mathbf{y}) \mathcal{T}(\mathrm{d} \mathbf{t})$-integrable by the transformation theorem. In particular, the linear term under the integral in (2.1) cancels. By combining (2.4) and (4.4), we see that $\int_{\mathbb{R}_{*}^{n}} e^{\mathrm{i}\langle\boldsymbol{\theta}, \mathbf{y}\rangle}-1 \mathcal{Y}(\mathrm{d} \mathbf{y})$ matches the integral in (4.5).

In view of (2.6) and (4.4), the Lévy density formula follows straightforwardly.

Remark 4.2. Strong univariate subordination of an arbitrary Brownian motion with an independent univariate Thorin subordinator was investigated in [11]. The corresponding class of Lévy processes was called $V G G^{n, 1}$ in [7]. Using our notation, we have $V G G^{n, 1}\left(d, \mu, \Sigma, \mathcal{U}_{0}\right):=$ $V G G^{n}\left(d \mathbf{e}, \boldsymbol{\mu}, \Sigma, \int_{(0, \infty)} \boldsymbol{\delta}_{u \mathbf{e}} \mathcal{U}_{0}(\mathrm{~d} u)\right)$, where $\boldsymbol{\mu} \in \mathbb{R}^{n}, d \in[0, \infty)$, while $\Sigma \in \mathbb{R}^{n \times n}$ is an arbitrary covariance matrix and $\mathcal{U}_{0}$ is a univariate Thorin measure. The $V G^{n}$-process [16] provides us with an example of a $V G G^{n, 1}$-process.

The $V G G^{n, n}$-class was introduced in [7] to complement the $V G G^{n, 1}$-class and contains processes formed by strong multivariate subordination of an independent-component Brownian motion with a Thorin subordinator. More specifically, $V G G^{n, n}(\mathbf{d}, \boldsymbol{\mu}, \Sigma, \mathcal{U}):=V G G^{n}(\mathbf{d}, \boldsymbol{\mu}, \Sigma, \mathcal{U})$ where $\mathbf{d} \in[0, \infty)^{n}, \boldsymbol{\mu} \in \mathbb{R}^{n}$, while $\Sigma$ is a covariance matrix of diagonal form and $\mathcal{U}$ is an $n$ dimensional Thorin measure. The strong $V \boldsymbol{\alpha} G$-process [23] is an example of a $V G G^{n, n}$-process.

In [7] (see Part (i) of their Theorems 2.3 and 2.5), formulae of the characteristic exponents of $V G G^{n, 1} \cup V G G^{n, n}$-processes are stated separately, while our Theorem 4.1 unifies both classes as special cases.

Remark 4.3. Though it does not need to be an element of the $V G G^{n, 1} \cup V G G^{n, n}$-class, a $W V \boldsymbol{\alpha} G$-process always belongs to the $V G G^{n}$-class.

If $Y \stackrel{D}{=} B \odot T \sim W V \boldsymbol{\alpha} G^{n}(a, b, \boldsymbol{\alpha}, \boldsymbol{\mu}, \Sigma)$ in (2.19), then $Y$ is also a $V G G^{n}$-process. More specifically, with $\mathcal{U}_{a, b, \alpha}$ as in Remark 4.1, we have

$$
Y \stackrel{D}{=} B \odot T \sim B M^{n}(\boldsymbol{\mu}, \Sigma) \odot G G C_{S}^{n}\left(\mathbf{0}, \mathcal{U}_{a, b, \boldsymbol{\alpha}}\right)=V G G^{n}\left(\mathbf{0}, \boldsymbol{\mu}, \Sigma, \mathcal{U}_{a, b, \boldsymbol{\alpha}}\right) .
$$

In particular, it follows from Theorem 4.1 that, $\boldsymbol{\theta}=\left(\theta_{1}, \ldots, \theta_{n}\right) \in \mathbb{R}^{n}$,

$$
\begin{aligned}
\Psi_{Y}(\boldsymbol{\theta})= & \Psi_{B \odot T}(\boldsymbol{\theta}) \\
= & -a \ln \left\{\left(b-\mathrm{i}\langle\boldsymbol{\alpha} \diamond \boldsymbol{\mu}, \boldsymbol{\theta}\rangle+\frac{1}{2}\|\boldsymbol{\theta}\|_{\boldsymbol{\alpha} \diamond \Sigma}^{2}\right) / b\right\} \\
& -\sum_{k=1}^{n} \beta_{k} \ln \left\{\left(b-\mathrm{i} \alpha_{k} \mu_{k} \theta_{k}+\frac{1}{2} \alpha_{k} \theta_{k}^{2} \Sigma_{k k}\right) / b\right\} .
\end{aligned}
$$

If, in addition, $\Sigma$ is invertible, then combining Theorem 4.1 and (2.6), and recalling $K_{1 / 2}(r)=$ $\pi^{1 / 2} e^{-r}(2 r)^{-1 / 2}, r>0$, (see [10], their Equation (8.469)-3), we find that $Y$ has Lévy measure $\mathcal{Y}$ given by

$$
\mathcal{Y}(\mathrm{d} \mathbf{y})=f_{0}(\mathbf{y}) \mathrm{d} \mathbf{y}+\sum_{k=1}^{n} \boldsymbol{\delta}_{0}^{\otimes(k-1)} \otimes\left(f_{k}\left(y_{k}\right) \mathrm{d} y_{k}\right) \otimes \boldsymbol{\delta}_{0}^{\otimes(n-k)}
$$


where $\mathbf{y}=\left(y_{1}, \ldots, y_{n}\right) \in \mathbb{R}_{*}^{n}$,

$$
f_{0}(\mathbf{y})=\frac{2 a \exp \left\{\langle\mathbf{y}, \boldsymbol{\alpha} \diamond \boldsymbol{\mu}\rangle_{(\boldsymbol{\alpha} \diamond \Sigma)^{-1}}\right\}}{(2 \pi)^{n / 2}|\boldsymbol{\alpha} \diamond \Sigma|^{1 / 2}\|\mathbf{y}\|_{(\boldsymbol{\alpha} \diamond \Sigma)^{-1}}^{n}} \mathfrak{K}_{n / 2}\left\{\|\mathbf{y}\|_{(\boldsymbol{\alpha} \diamond \Sigma)^{-1}}\left(2 b+\|\boldsymbol{\alpha} \diamond \boldsymbol{\mu}\|_{(\boldsymbol{\alpha} \diamond \Sigma)^{-1}}^{2}\right)^{1 / 2}\right\},
$$

and $1 \leq k \leq n, y \in \mathbb{R}_{*}$,

$$
f_{k}(y)=\frac{\beta_{k}}{|y|} \exp \left\{\left(\alpha_{k}^{1 / 2} \mu_{k} y-|y|\left(2 b \Sigma_{k k}+\alpha_{k} \mu_{k}^{2}\right)^{1 / 2}\right) /\left(\alpha_{k}^{1 / 2} \Sigma_{k k}\right)\right\} .
$$

Alternatively, this decomposition could be derived from Remark 3.9.

Sample Paths. To see how sample path properties such as $q$-variation of the Thorin subordinator is propagated through Brownian motion, we generalise the corresponding result in [7] (see their Propositions 2.1-2.2; see Section 5.4 for a proof).

Proposition 4.1. Let $T \sim G G C_{S}^{n}(\mathbf{d}, \mathcal{U})$ and $Y \sim V G G^{n}(\mathbf{d}, \boldsymbol{\mu}, \Sigma, \mathcal{U})$ with Lévy measures $\mathcal{T}$ and $\mathcal{Y}$, respectively. Suppose $0<q<1$.

(i) $\int_{\mathbb{D}_{*}^{C}} \mathcal{U}(\mathrm{d} \mathbf{u}) /\|\mathbf{u}\|^{q}$ is finite if and only if $\int_{\mathbb{D}_{*}}\|\mathbf{t}\|^{q} \mathcal{T}(\mathrm{d} \mathbf{t})$ is.

(ii) If $\int_{\mathbb{D} C} \mathcal{U}(\mathrm{du}) /\|\mathbf{u}\|^{q}$ is finite, then $\int_{\mathbb{D}_{*}}\|\mathbf{y}\|^{2 q} \mathcal{Y}(\mathrm{d} \mathbf{y})$ is finite. If $\Sigma$ is invertible, then also the converse holds.

(iii) If $\mathbf{d}=\mathbf{0}$ and $\int_{\mathbb{D} C} \mathcal{U}(\mathrm{du}) /\|\mathbf{u}\|^{1 / 2}$ is finite, then $Y$ is a drift-less $F V^{n}$-process. If, in addition, $\Sigma$ is invertible, then $Y \sim F V^{n}$ implies $\mathbf{d}=\mathbf{0}$ and the finiteness of $\int_{\mathbb{D} C} \mathcal{U}(\mathrm{du}) /\|\mathbf{u}\|^{1 / 2}$.

Remark 4.4. In [7] (see their Remarks 2.8-2.9), examples are found of drift-less multivariate Thorin subordinators subordinating Brownian motion in the strong sense, with the associated $V G G^{n, 1} \cup V G G^{n, n}$-process having sample paths of unbounded variation. Proposition 4.1 states that those examples have counterparts in the weak sense.

Remark 4.5. If $\mathcal{U}$ is a finitely supported nonnegative measure on $[0, \infty)_{*}^{n}$, then $\mathcal{U}$ is in particular a Thorin measure, and any associated drift-less $V G G^{n}$-process must be a $F V^{n}$-process as is straightforwardly derived from Proposition 4.1(iii). In particular, see Remark 4.1, weak variance$\boldsymbol{\alpha}$-gamma processes are drift-less $F V^{n}$-processes.

Remark 4.6. Weak subordination has applications in financial modelling. In [18], log returns of multiple dependent prices and $V G$-marginals were modelled using a $W V \boldsymbol{\alpha} G^{n}$-process. In [17], the $\log$ returns were modelled using a weakly subordinated process where the subordinator, interpreted as an information flow process, has jump dependence specified by a Lévy copula while its marginals may be chosen arbitrarily. In [15], $W V \boldsymbol{\alpha} G^{n}$-processes were applied to instantaneous portfolio theory. In our future work, we will consider statistical inference for $W V \boldsymbol{\alpha} G^{n}$ processes [8], and conditions for the self-decomposability within the weak $V G G^{n}$-class [9]. 


\section{Proofs}

\subsection{Proof of Proposition 2.1}

For $\mathbf{t}=\left(t_{1}, \ldots, t_{n}\right) \in[0, \infty)^{n}$ recall that $\langle(1), \ldots,(n)\rangle$ denotes the associated permutation of the ordering $t_{(1)} \leq \cdots \leq t_{(n)}$ and $\Delta t_{(k)}$ correspond to its $k$ th spacing. For $1 \leq m \leq n$, let $\boldsymbol{\pi}_{m}:=\boldsymbol{\pi}_{\{(m), \ldots,(n)\}}: \mathbb{R}^{n} \rightarrow \mathbb{R}^{n}, \boldsymbol{\theta} \mapsto \boldsymbol{\theta} \boldsymbol{\pi}_{m}$. Let $X \sim L^{n}(\boldsymbol{\mu}, \Sigma, \mathcal{X})$ with $\Psi$ as in (2.1). For $\boldsymbol{\theta}=\left(\theta_{1}, \ldots, \theta_{n}\right) \in \mathbb{R}^{n}$, we have

$$
\sum_{k=1}^{n} \theta_{k} X_{k}\left(t_{k}\right)=\sum_{k=1}^{n} \theta_{(k)} X_{(k)}\left(t_{(k)}\right)=\sum_{k=1}^{n} \sum_{m=1}^{k} \theta_{(k)}\left(X_{(k)}\left(t_{(m)}\right)-X_{(k)}\left(t_{(m-1)}\right)\right),
$$

and thus, by interchanging the order of summation on the RHS,

$$
\sum_{k=1}^{n} \theta_{k} X_{k}\left(t_{k}\right)=\sum_{m=1}^{n} \sum_{k=m}^{n} \theta_{(k)}\left(X_{(k)}\left(t_{(m)}\right)-X_{(k)}\left(t_{(m-1)}\right)\right),
$$

giving $\mathbb{E} \exp (\mathrm{i}\langle\boldsymbol{\theta}, X(\mathbf{t})\rangle)=\exp \left\{\sum_{m=1}^{n} \Delta t_{(m)} \Psi\left(\boldsymbol{\theta} \boldsymbol{\pi}_{m}\right)\right\}$ which matches (2.10).

Since projections are self-adjoint, we must have

$$
\Psi\left(\boldsymbol{\theta} \boldsymbol{\pi}_{m}\right)=\mathrm{i}\left\langle\boldsymbol{\mu} \boldsymbol{\pi}_{m}, \boldsymbol{\theta}\right\rangle-\frac{1}{2}\left\|\boldsymbol{\theta} \boldsymbol{\pi}_{m}\right\|_{\Sigma}^{2}+\int_{\mathbb{R}_{*}^{n}}\left(e^{\mathrm{i}\left\langle\boldsymbol{\theta}, \mathbf{x} \boldsymbol{\pi}_{m}\right\rangle}-1-\mathrm{i}\left\langle\boldsymbol{\theta}, \mathbf{x} \boldsymbol{\pi}_{m}\right\rangle \mathbf{1}_{\mathbb{D}}(\mathbf{x})\right) \mathcal{X}(\mathrm{d} \mathbf{x}) .
$$

As $\langle\mathbf{t} \diamond \boldsymbol{\mu}, \boldsymbol{\theta}\rangle=\sum_{m=1}^{n} \Delta t_{(m)}\left\langle\boldsymbol{\mu} \boldsymbol{\pi}_{m}, \boldsymbol{\theta}\right\rangle$ and $\|\boldsymbol{\theta}\|_{\mathbf{t} \diamond \Sigma}^{2}=\sum_{m=1}^{n} \Delta t_{(m)}\left\|\boldsymbol{\theta} \boldsymbol{\pi}_{m}\right\|_{\Sigma}^{2}$, we get by recalling (2.8) and $\mathbf{1}_{\mathbb{D}} \circ \boldsymbol{\pi}_{m}-\mathbf{1}_{\mathbb{D}}=\left(\mathbf{1}_{\mathbb{D} C}\right)\left(\mathbf{1}_{\mathbb{D}} \circ \boldsymbol{\pi}_{m}\right)$ that

$$
\begin{aligned}
& \sum_{m=1}^{n} \Delta t_{(m)} \int_{\mathbb{R}_{*}^{n}}\left(\exp \left\{\mathrm{i}\left\langle\boldsymbol{\theta}, \boldsymbol{\pi}_{m}(\mathbf{x})\right\rangle\right\}-1-\mathrm{i}\left\langle\boldsymbol{\theta}, \boldsymbol{\pi}_{m}(\mathbf{x})\right\rangle \mathbf{1}_{\mathbb{D}}(\mathbf{x})\right) \mathcal{X}(\mathrm{d} \mathbf{x}) \\
& \quad=\mathrm{i}\langle\mathbf{c}(\mathbf{t}, \mathcal{X}), \boldsymbol{\theta}\rangle+\int_{\mathbb{R}_{*}^{n}}\left(\exp \{\mathrm{i}\langle\boldsymbol{\theta}, \mathbf{x}\rangle\}-1-\mathrm{i}\langle\boldsymbol{\theta}, \mathbf{x}\rangle \mathbf{1}_{\mathbb{D}}(\mathbf{x})\right)(\mathbf{t} \diamond \mathcal{X})(\mathrm{d} \mathbf{x}) .
\end{aligned}
$$

By combining the above, (2.11) follows from (2.10), completing the proof.

\subsection{Proof of Theorem 2.1}

We collect some useful estimates into a lemma. Its proof and purpose follow [21] (see his Lemma 30.3) and [3] (see the proof of their Theorem 3.2). However, we have to adapt these results to deal with the multivariate time parameter.

Lemma 5.1. If $X \sim L^{n}(\boldsymbol{\mu}, \Sigma, \mathcal{X})$ and $\boldsymbol{\theta} \in \mathbb{R}^{n}$, then there exist finite $C_{1}=C_{1}(\boldsymbol{\theta}, X), C_{2}=C_{2}(X)$ and $C_{3}=C_{3}(X)$ such that, for $\mathbf{t} \in[0, \infty)^{n}$,

$$
\left|\Phi_{X(\mathbf{t})}(\boldsymbol{\theta})-1\right| \leq C_{1}(1 \wedge\|\mathbf{t}\|),
$$




$$
\begin{aligned}
\mathbb{E}\left[1 \wedge\|X(\mathbf{t})\|^{2}\right] & \leq C_{2}(1 \wedge\|\mathbf{t}\|), \\
\mathbb{E}[1 \wedge\|X(\mathbf{t})\|] & \leq C_{2}^{1 / 2}\left(1 \wedge\|\mathbf{t}\|^{1 / 2}\right), \\
\left\|\mathbb{E}\left[X(\mathbf{t}) \mathbf{1}_{\mathbb{D}}(X(\mathbf{t}))\right]\right\| & \leq C_{3}(1 \wedge\|\mathbf{t}\|) .
\end{aligned}
$$

Proof. Let $\mathbf{t}=\left(t_{1}, \ldots, t_{n}\right) \in[0, \infty)^{n}$ and $\boldsymbol{\theta}=\left(\theta_{1}, \ldots, \theta_{n}\right) \in \mathbb{R}^{n}$, and introduce a Lévy measure $\mathcal{N}:=\sum_{\langle(1), \ldots,(n)\rangle} \sum_{k=1}^{n} \mathcal{X}_{\{(k), \ldots,(n)\}}$ with the first summation taken over all permutations $\langle(1), \ldots,(n)\rangle$.

Recall $\left|e^{z}-1\right| \leq|z|$, holds for $z \in \mathbb{C}$ with $\Re z \leq 0$, and, in particular, for $z:=\mathbf{t} \diamond \Psi(\boldsymbol{\theta})$ in (2.11). Further, we have $|\Re(\mathbf{t} \diamond \Psi(\boldsymbol{\theta}))| \leq C_{11}\|\mathbf{t}\|$, where

$$
C_{11}:=\frac{1}{2} \sum_{k, l=1}^{n}\left|\theta_{k} \theta_{l} \Sigma_{k l}\right|+\int_{\mathbb{R}_{*}^{n}}|1-\cos \langle\boldsymbol{\theta}, \mathbf{x}\rangle| \mathcal{N}(\mathrm{d} \mathbf{x}) .
$$

In (2.9), note $\|\mathbf{c}(\mathbf{t}, \mathcal{X})\| \leq n \mathcal{X}\left(\mathbb{D}^{C}\right)\|\mathbf{t}\|$, giving $|\Im(\mathbf{t} \diamond \Psi(\boldsymbol{\theta}))| \leq C_{12}\|\mathbf{t}\|$, where

$$
C_{12}:=n\left(\|\boldsymbol{\mu}\|+n \mathcal{X}\left(\mathbb{D}^{C}\right)\right)\|\boldsymbol{\theta}\|+\int_{\mathbb{R}_{*}^{n}}\left|\langle\boldsymbol{\theta}, \mathbf{x}\rangle \mathbf{1}_{\mathbb{D}}(\mathbf{x})-\sin \langle\boldsymbol{\theta}, \mathbf{x}\rangle\right| \mathcal{N}(\mathrm{d} \mathbf{x}) .
$$

Plainly, $C_{11}$ and $C_{12}$ are finite constants in view of by (2.2). Choosing $C_{13}^{2}:=C_{13}^{2}(\boldsymbol{\theta}):=C_{11}^{2}+$ $C_{12}^{2}$ shows $\left|\Phi_{X(\mathbf{t})}(\boldsymbol{\theta})-1\right| \leq C_{13}\|\mathbf{t}\|$, so that (5.1) holds for some finite $C_{1}=C_{1}(\boldsymbol{\theta})$.

Setting $\mathcal{Y}_{\mathbf{t}}(A):=(\mathbf{t} \diamond \mathcal{X})\left(A \cap \mathbb{D}^{C}\right)$ and $\mathcal{Z}_{\mathbf{t}}(A):=(\mathbf{t} \diamond \mathcal{X})(A \cap \mathbb{D}), A \subseteq \mathbb{R}_{*}^{n}$ Borel, yields Lévy measures $\mathcal{Y}_{\mathbf{t}}$ and $\mathcal{Z}_{\mathbf{t}}$ on $\mathbb{R}_{*}^{n}$ with disjoint supports and associated independent Lévy processes $Y^{(\mathbf{t})} \sim L^{n}\left(\mathbf{0}, 0, \mathcal{Y}_{\mathbf{t}}\right)$ and $Z^{(\mathbf{t})}=\left(Z_{1}^{(\mathbf{t})}, \ldots, Z_{n}^{(\mathbf{t})}\right) \sim L^{n}\left(\mathbf{t} \diamond \boldsymbol{\mu}+\mathbf{c}(\mathbf{t}, \mathcal{X}), \mathbf{t} \diamond \Sigma, \mathcal{Z}_{\mathbf{t}}\right)$, respectively.

By Proposition 2.1, we may decompose $X(\mathbf{t}) \stackrel{D}{=} Y^{(\mathbf{t})}(1)+Z^{(\mathbf{t})}(1)$ into a sum of independent $n$-dimensional random vectors.

Note $Y^{(\mathbf{t})}$ is a compound Poisson process with jumps in $\|\cdot\|$-modulus larger than 1 . In particular, $\left\{Y^{(\mathbf{t})}\right.$ has no jumps in time interval $\left.[0,1]\right\} \subseteq\left\{Y^{(\mathbf{t})}(1)=0\right\}$, giving the bound

$$
\mathbb{P}\left(Y^{(\mathbf{t})}(1) \neq 0\right) \leq 1-\mathbb{P}\left(Y^{(\mathbf{t})} \text { has no jumps in time interval }[0,1]\right)=1-\exp \left(-(\mathbf{t} \diamond \mathcal{X})\left(\mathbb{D}^{C}\right)\right) .
$$

Since $(\mathbf{t} \diamond \mathcal{X})\left(\mathbb{D}^{C}\right) \leq\|\mathbf{t}\| \mathcal{N}\left(\mathbb{D}^{C}\right)$ and $1-e^{-x} \leq x, x \in \mathbb{R}$, we have

$$
\mathbb{P}\left(Y^{(\mathbf{t})}(1) \neq 0\right) \leq \mathcal{N}\left(\mathbb{D}^{C}\right)\|\mathbf{t}\|
$$

On the other hand, $Z^{(\mathbf{t})}$ has jumps bounded in norm by 1 . In particular, $Z^{(\mathbf{t})}(1)$ has finite moments of all order. Recall $\mathbb{E}\left[Z_{k}^{(\mathbf{t})}(1)\right]=\mu_{k} t_{k}+c_{k}(\mathbf{t}, \mathcal{X})$ and $\operatorname{Var}\left(Z_{k}^{(\mathbf{t})}(1)\right)=\Sigma_{k k} t_{k}+\int_{\mathbb{D}_{*}} x_{k}^{2}(\mathbf{t} \diamond \mathcal{X})(\mathrm{d} \mathbf{x})$ for $1 \leq k \leq n$ (see [21], his Example 25.12).

By (2.2), $\left.C_{21}:=2\|\boldsymbol{\mu}\|^{2}+2 n^{2} \mathcal{X}\left(\mathbb{D}^{C}\right)^{2}+\operatorname{trace}(\Sigma)+\int_{\mathbb{D}_{*}}\|\mathbf{x}\|^{2} \mathcal{N}(\mathrm{d} \mathbf{x})\right\}$ is a finite constant, in addition satisfying

$$
\mathbb{E}\left[\left\|Z^{(\mathbf{t})}(1)\right\|^{2}\right] \leq C_{21}\left(\|\mathbf{t}\|+\|\mathbf{t}\|^{2}\right)
$$


By (5.5)-(5.6), $\mathbb{E}\left[1 \wedge\|X(\mathbf{t})\|^{2}\right] \leq C_{22}\left(\|\mathbf{t}\|+\|\mathbf{t}\|^{2}\right)$ holds with the choice $C_{22}:=\mathcal{N}\left(\mathbb{D}^{C}\right)+C_{21}$, by noting $\mathbb{E}\left[1 \wedge\|X(\mathbf{t})\|^{2}\right] \leq \mathbb{P}\left(Y^{(\mathbf{t})}(1) \neq \mathbf{0}\right)+\mathbb{E}\left[\left\|Z^{(\mathbf{t})}(1)\right\|^{2}\right]$. This completes the proof of (5.2), while (5.3) is implied by (5.2) and the Cauchy-Schwarz inequality.

Recall $\|\mathbf{z}\|_{\infty}^{2}:=\max _{1 \leq k \leq n}\left|z_{k}\right|^{2} \leq\|\mathbf{z}\|:=\mathbf{z}^{\prime}, \mathbf{z}=\left(z_{1}, \ldots, z_{n}\right) \in \mathbb{C}^{n}$, and set $\mathbb{D}_{\infty}:=\left\{\mathbf{x} \in \mathbb{R}^{n}:\right.$ $\left.\|\mathbf{x}\|_{\infty} \leq 1\right\}$. If $g(x):=e^{\mathrm{i} x}-1, x \in \mathbb{R}$, we have

$$
\begin{aligned}
\left\|\mathbb{E}\left[X(\mathbf{t}) \mathbf{1}_{\mathbb{D}_{\infty}}(X(\mathbf{t}))\right]\right\|_{\infty} \leq & \max _{1 \leq j \leq n}\left|\mathbb{E}\left[g\left(X_{j}(\mathbf{t})\right) \mathbf{1}_{\mathbb{D}_{\infty}^{C}}(X(\mathbf{t}))\right]\right| \\
& +\max _{1 \leq j \leq n}\left|\mathbb{E}\left[\left(g\left(X_{j}(\mathbf{t})\right)-\mathrm{i} X_{j}(\mathbf{t})\right) \mathbf{1}_{\mathbb{D}_{\infty}}(X(\mathbf{t}))\right]\right| \\
& +\max _{1 \leq j \leq n}\left|\mathbb{E}\left[g\left(X_{j}(\mathbf{t})\right)\right]\right| .
\end{aligned}
$$

By noting $\mathbf{1}_{\mathbb{D}_{\infty}^{C}} \leq \mathbf{1}_{\mathbb{D}^{C}} \leq 1 \wedge\|\cdot\|^{2}$, we get

$$
\left|\mathbb{E}\left[g\left(X_{j}(\mathbf{t})\right) \mathbf{1}_{\mathbb{D}_{\infty}^{C}}(X(\mathbf{t}))\right]\right| \leq 2 \mathbb{E}\left[\mathbf{1}_{\mathbb{D}_{\infty}^{C}}(X(\mathbf{t}))\right] \leq 2 \mathbb{E}\left[1 \wedge\|X(\mathbf{t})\|^{2}\right], \quad 1 \leq j \leq n,
$$

and then (5.2) can be applied. Next, by noting $4|g(x)-i x|^{2} \leq x^{4}+x^{6}, x \in \mathbb{R}$, we get

$$
\left|\mathbb{E}\left[\left(g\left(X_{j}(\mathbf{t})\right)-\mathrm{i} X_{j}(\mathbf{t})\right) \mathbf{1}_{\mathbb{D}_{\infty}}(X(\mathbf{t}))\right]\right| \leq \mathbb{E}\left[1 \wedge X_{j}^{2}(\mathbf{t})\right] \leq \mathbb{E}\left[1 \wedge\|X(\mathbf{t})\|^{2}\right], \quad 1 \leq j \leq n,
$$

and then (5.2) can be applied. Lastly, we get $\left|\mathbb{E}\left[g\left(X_{j}(\mathbf{t})\right)\right]\right|=\left|\Phi_{X(\mathbf{t})}\left(\mathbf{e}_{j}\right)-1\right|, 1 \leq j \leq n$, and then (5.1) can be applied with $\boldsymbol{\theta} \in\left\{\mathbf{e}_{1}, \ldots, \mathbf{e}_{n}\right\}$.

Combining the above yields $\left\|\mathbb{E}\left[X(\mathbf{t}) \mathbf{1}_{\mathbb{D}_{\infty}}(X(\mathbf{t}))\right]\right\|_{\infty} \leq C_{31}(1 \wedge\|\mathbf{t}\|)$ for some finite constant $C_{31}$. Applying the Euclidean triangle inequality and $\|\cdot\| \leq n^{1 / 2}\|\cdot\|_{\infty}$ yields

$$
\left\|\mathbb{E}\left[X(\mathbf{t}) \mathbf{1}_{\mathbb{D}}(X(\mathbf{t}))\right]\right\| \leq n^{1 / 2}\left\|\mathbb{E}\left[X(\mathbf{t}) \mathbf{1}_{\mathbb{D}_{\infty}}(X(\mathbf{t}))\right]\right\|_{\infty}+\left\|\mathbb{E}\left[X(\mathbf{t}) \mathbf{1}_{\mathbb{D}_{\infty} \backslash \mathbb{D}}(X(\mathbf{t}))\right]\right\| .
$$

The second term on the RHS is bounded from above by $n^{1 / 2} \mathbb{E}\left[\mathbf{1}_{\mathbb{D}}(X(\mathbf{t}))\right]$, and we found this to be bounded from above by $n^{1 / 2} \mathbb{E}\left[1 \wedge\|X(\mathbf{t})\|^{2}\right]$, to which (5.2) was applicable. This completes the proof of the lemma.

Proof of Theorem 2.1(i). Plainly, $\Theta$ in (2.14) is a valid covariance matrix as $\mathbf{d} \diamond \Sigma$ is the covariance matrix of $B(\mathbf{d})$ with $B \sim B M^{n}(0, \Sigma)$. It remains to validate that $\mathcal{Z}$ in (2.15) is a Lévy measure. By (2.11), if $\boldsymbol{\theta} \in \mathbb{R}^{n}$, then $\mathbf{t} \mapsto(\mathbf{t} \diamond \Psi)(\boldsymbol{\theta})$ is a continuous function with domain $\mathbf{t} \in[0, \infty)^{n}$. In particular, the family of probability measures $\left\{\mathbb{P}(X(\mathbf{t}) \in \mathrm{d} \mathbf{x}): \mathbf{t} \in[0, \infty)^{n}\right\}$ is weakly continuous, and $\mathbb{P}(X(\mathbf{t}) \in \mathrm{d} \mathbf{x})$ is a Markov kernel from $[0, \infty)^{n}$ to $\mathbb{R}^{n}$, and $\mathcal{Z}_{0}(\mathrm{~d} \mathbf{t}$, dx $):=$ $\mathbf{1}_{[0, \infty)_{*}^{n} \times \mathbb{R}^{n}}(\mathbf{t}, \mathbf{x}) \mathbb{P}(X(\mathbf{t}) \in \mathrm{d} \mathbf{x}) \mathcal{T}(\mathrm{d} \mathbf{t})$ is a well-defined $\sigma$-finite Borel measure on the punctured product $\left([0, \infty)^{n} \times \mathbb{R}^{n}\right)_{*}$, for which we note

$$
\int_{\left([0, \infty)^{n} \times \mathbb{R}^{n}\right)_{*}} 1 \wedge\|(\mathbf{t}, \mathbf{x})\|^{2} \mathcal{Z}_{0}(\mathrm{~d} \mathbf{t}, \mathrm{d} \mathbf{x})=\int_{[0, \infty)_{*}^{n}} \mathbb{E}\left[1 \wedge\|(I \mathbf{e}, X)(\mathbf{t})\|^{2}\right] \mathcal{T}(\mathrm{d} \mathbf{t}),
$$

where $\mathbf{e}=(1, \ldots, 1) \in \mathbb{R}^{n}$ and $(I \mathbf{e}, X)$ is an augmented $2 n$-dimensional Lévy process. 
For $\mathbf{t} \in[0, \infty)^{n}$, by noting $\|(\mathbf{t}, \mathbf{t})\|^{2}=2\|\mathbf{t}\|^{2}$ and $1 \wedge\|(\mathbf{t}, \mathbf{t})\| \leq 2^{1 / 2}(1 \wedge\|\mathbf{t}\|), \mathbf{t} \in[0, \infty)^{n}$, and applying (5.2) with $C_{2}:=C_{2}((I \mathbf{e}, X))$, we get

$$
\mathbb{E}\left[1 \wedge\|(I \mathbf{e}, X)(\mathbf{t})\|^{2}\right] \leq C_{2}(1 \wedge\|(\mathbf{t}, \mathbf{t})\|) \leq 2^{1 / 2} C_{2}(1 \wedge\|\mathbf{t}\|) .
$$

As (2.3) holds for $\mathcal{T}$, the RHS in the last display is $\mathcal{T}$-integrable, hence $\mathcal{Z}_{0}$ and $\mathcal{Z}$ in (2.15) are Lévy measures by (5.7).

Note $\|\mathbf{t}\| \mathbb{P}((\mathbf{t}, X(\mathbf{t})) \in \mathbb{D}) \leq\|\mathbf{t}\| \mathbf{1}_{\mathbb{D}}(\mathbf{t})$ for all $\mathbf{t} \in[0, \infty)^{n}$. As the RHS is $\mathcal{T}$-integrable by (2.3), so is the LHS, and then (2.12) is well-defined. The RHS of (2.13) is well-defined as an implication of (5.4), applied to the augmented process ( $I \mathbf{e}, X)$.

Proof of Theorem 2.1(ii) On a suitable augmentation of $(\Omega, \mathcal{F}, \mathbb{P})$, where $T$ lives, we find $W \sim L^{2 n}\left(\mathbf{m}, \Theta, \boldsymbol{\delta}_{\mathbf{0}} \otimes(\mathbf{d} \diamond \mathcal{X})\right), \mathbf{m}=\left(\mathbf{m}_{1}, \mathbf{m}_{2}\right)$ with $\mathbf{m}_{1}, \mathbf{m}_{2}$ and $\Theta$ as in (2.12)- (2.14), and a family $\xi=\left\{\xi(t, \mathbf{t}):(t, \mathbf{t}) \in[0, \infty) \times[0, \infty)_{*}^{n}\right\}$ of independent random vectors, satisfying $\xi(t, \mathbf{t}) \stackrel{D}{=} X(\mathbf{t})$ for $(t, \mathbf{t}) \in[0, \infty) \times[0, \infty)_{*}^{n}$, such that $T, \xi, W$ are independent. Introduce a marked Poisson point process

$$
\mathbb{Z}_{0}:=\sum_{t>0} \boldsymbol{\delta}_{(t, T(t)-T(t-), \xi(t, T(t)-T(t-)))},
$$

thus being a Poisson point process with intensity $\mathrm{d} t \otimes \mathcal{Z}_{0}$, where $\mathcal{Z}_{0}$ is the Lévy measure in Part (i). Particularly, $\mathbb{Z}_{0}$ is the point measure of jumps of a Lévy process $Z_{0} \sim L^{2 n}\left(\mathbf{0}, \mathbf{0}, \mathcal{Z}_{0}\right)$ via its Lévy-Itô decomposition. As $Z_{1}=T, Z=\left(Z_{1}, Z_{2}\right):=Z_{0}+W \stackrel{D}{=}(T, X \odot T)$ is $T$ subordinating $X$ in the semi-strong sense.

Proof of Theorem 2.1(iii) If, in addition, $\int_{[0,1]_{*}^{n}}\|\mathbf{t}\|^{1 / 2} \mathcal{T}(\mathrm{d} \mathbf{t})$ is finite, then (2.3) holds. This follows similarly as in (5.7), but using (5.3) instead of (5.2).

\subsection{Proof of Propositions 3.9 and 3.10}

Let $T=\left(T_{1}, T_{2}\right) \sim S^{2}$ and $X=\left(X_{1}, X_{2}\right) \sim L^{2}$ be independent. For $\boldsymbol{\theta}=\left(\theta_{1}, \theta_{2}\right) \in \mathbb{R}^{2}, t \geq s \geq 0$, introduce $\widehat{\Psi}_{X}(\boldsymbol{\theta}):=\Psi_{X}(\boldsymbol{\theta})-\Psi_{X_{1}}\left(\theta_{1}\right)-\Psi_{X_{2}}\left(\theta_{2}\right)$.

Proof of Propositions 3.9. For $\boldsymbol{\theta}=\left(\theta_{1}, \theta_{2}\right) \in \mathbb{R}^{2}, t \geq s \geq 0$, introduce $A(s, t):=\left(T_{1}(s) \wedge\right.$ $\left.T_{2}(t)\right)-\left(T_{1}(s) \wedge T_{2}(s)\right)$ and $Z(s, t, \boldsymbol{\theta}):=T_{1}(s) \Psi_{X_{1}}\left(\theta_{1}\right)+\left(T_{2}(t)-T_{2}(s)\right) \Psi_{X_{2}}\left(\theta_{2}\right)$.

In view of (2.10), for $\boldsymbol{\theta}=\left(\theta_{1}, \theta_{2}\right) \in \mathbb{R}^{2}, r \geq 0, t \geq s \geq 0$, note

$$
(r, t, s) \diamond \Psi_{X_{1}, X_{2}, X_{2}}\left(\boldsymbol{\theta},-\theta_{2}\right)=r \Psi_{X_{1}}\left(\theta_{1}\right)+(t-s) \Psi_{X_{2}}\left(\theta_{2}\right)+\widehat{\Psi}_{X}(\boldsymbol{\theta})(r \wedge t-r \wedge s),
$$

and thus, by conditioning on $T$,

$$
\Phi_{\left(X_{1}\left(T_{1}(s)\right), X_{2}\left(T_{2}(t)\right)-X_{2}\left(T_{2}(s)\right)\right)}(\boldsymbol{\theta})=\mathbb{E} \exp \left\{Z(s, t, \boldsymbol{\theta})+\widehat{\Psi}_{X}(\boldsymbol{\theta}) A(s, t)\right\} .
$$

As $X \circ T$ is assumed to be a Lévy process, both $T$ and $X \circ T$, have independent increments across the components. Conditioning the LHS of the last display on $T$ shows the following identity, for $\boldsymbol{\theta}=\left(\theta_{1}, \theta_{2}\right) \in \mathbb{R}^{2}, t \geq s \geq 0$,

$$
\mathbb{E} \exp \{Z(s, t, \boldsymbol{\theta})\}=\mathbb{E} \exp \left\{Z(s, t, \boldsymbol{\theta})+\widehat{\Psi}_{X}(\boldsymbol{\theta}) A(s, t)\right\} .
$$


(i) Assume $X \stackrel{D}{=}-X$. Since $X_{1}$ and $X_{2}$ are dependent, there exist $\boldsymbol{\theta}=\left(\theta_{1}, \theta_{2}\right) \in \mathbb{R}^{2}$ such that $\widehat{\Psi}_{X}(\boldsymbol{\theta}) \neq 0$. By symmetry, $\Psi_{X}(\boldsymbol{\theta}), \widehat{\Psi}_{X}(\boldsymbol{\theta}), \Psi_{X_{j}}\left(\theta_{j}\right) \in \mathbb{R}, j=1,2$. Let $t>0, u \geq 1$. In (5.8) we have $Z(t, u t, \boldsymbol{\theta}) \in \mathbb{R}$, forcing $A(t, u t)=0$ almost surely. In particular, $u \mapsto A(t, u t)$ degenerates to the null process. As $T_{2}$ cannot degenerate to the null process, we must have $T_{2}(t)<T_{2}(u t)$ for some $u>1$ with probability one, and thus, $T_{1}(t) \leq T_{2}(t)$ almost surely. Reversing the role of $T_{1}$ and $T_{2}$ completes the proof of Part (i).

(ii) As $X_{1}, X_{2}$ are dependent we have $\widehat{\Psi}_{X}(\boldsymbol{\theta}) \neq 0$ for some $\boldsymbol{\theta} \in \mathbb{R}^{2}$. If $T$ is deterministic with drift $\left(d_{1}, d_{2}\right)$, then $\widehat{\Psi}_{X}(\boldsymbol{\theta}) A(t,(1+\varepsilon) t) \in 2 \pi \mathrm{i} \mathbb{Z}$ for $t, \varepsilon>0$, as an implication of (5.8), giving $d_{1} \leq d_{2}$, with the argument being completed as in (i).

(iii) Assume $T(1)$, and thus $A(t, 2 t)$ for all $t \geq 0$, admits a finite first moment. In addition, suppose there exists a sequence $\boldsymbol{\theta}_{n} \rightarrow \mathbf{0}$ as $n \rightarrow \infty$ such that $\widehat{\Psi}\left(\boldsymbol{\theta}_{n}\right) \neq 0$ and $\Re \widehat{\Psi}\left(\boldsymbol{\theta}_{n}\right) \leq 0$ for all $n$. As $\left|1-e^{z}\right| \leq|z|$ for $\Re z \leq 0$, note $\mid Z\left(t, 2 t, \boldsymbol{\theta}_{n}\right)\left(1-\exp \left\{A(t, 2 t) \widehat{\Psi}_{X}\left(\boldsymbol{\theta}_{n}\right)\right\}\right) / \widehat{\Psi}_{\left(\boldsymbol{\theta}_{n}\right) \mid \leq}$ $A(t, 2 t)$, and dominated convergence is applicable to (5.8), giving $A(t, 2 t)=0$ almost surely, since

$$
0=\lim _{n \rightarrow \infty} \mathbb{E}\left[Z\left(t, 2 t, \boldsymbol{\theta}_{n}\right)\left(1-\exp \left\{A(t, 2 t) \widehat{\Psi}_{X}\left(\boldsymbol{\theta}_{n}\right)\right\}\right) / \widehat{\Psi}_{X}\left(\boldsymbol{\theta}_{n}\right)\right]=\mathbb{E}[A(t, 2 t)]
$$

If $X(1)$ admits a finite second moment, then $\widehat{\Psi}_{X}(\boldsymbol{\theta})=-\rho \theta_{1} \theta_{2}+o\left(\|\boldsymbol{\theta}\|^{2}\right)$ as $\boldsymbol{\theta} \rightarrow \mathbf{0}$, where $\rho=$ $\operatorname{Cov}\left(X_{1}(1), X_{2}(1)\right)$, the existence of sequence as required in the previous paragraph is obvious, provided $\rho \neq 0$.

Proof of Proposition 3.10. Introduce $D:=T_{2}-T_{1} \sim F V^{1}(d, \mathcal{D})$. If $\boldsymbol{\theta}=\left(\theta_{1}, \theta_{2}\right) \in \mathbb{R}^{2}$, note $(r, s) \diamond \Psi_{X}(\boldsymbol{\theta})=(r \wedge s) \Psi_{X}(\boldsymbol{\theta})+(s-r)^{+} \Psi_{X_{2}}\left(\theta_{2}\right)+(s-r)^{-} \Psi_{X_{1}}\left(\theta_{1}\right), r, s \geq 0$, so that, by conditioning on $T$,

$$
\Phi_{X(T(t))}(\boldsymbol{\theta})=\mathbb{E}\left[\exp \left\{\left(T_{1}(t) \wedge T_{2}(t)\right) \Psi_{X}(\boldsymbol{\theta})+D^{+}(t) \Psi_{X_{2}}\left(\theta_{2}\right)+D^{-}(t) \Psi_{X_{1}}\left(\theta_{1}\right)\right\}\right]
$$

(i) Recall $T$ is monotonic if and only if either $D$ or $-D$ is a subordinator. As we assumed $T$ to have non-monotonic and non-deterministic components, one of the following exclusive cases holds (see [21], his Corollary 24.8 and his Theorem 24.10):

(a) $\mathcal{D}(-\infty, 0)>0, \mathcal{D}(0, \infty)=0$ and $d>0$, so that the support of the distribution of $D(1)$ is unbounded towards $-\infty$ with $d$ as its supremum;

(b) $\mathcal{D}(-\infty, 0)=0, \mathcal{D}(0, \infty)>0$ and $d<0$, so that the support of $D(1)$ is unbounded towards $\infty$ with $d$ as its infimum;

(c) $\mathcal{D}(-\infty, 0)>0$ and $\mathcal{D}(0, \infty)>0(d \in \mathbb{R}$ is arbitrary), so that the support of $D(1)$ is unbounded towards $\infty$ and $-\infty$.

In all cases, we have $\mathbb{P}(D(1)>0)>0$ and $\mathbb{P}(D(1)<0)>0$, implying $\mathbb{E}\left[D^{+}(1)\right]>0$ and $\mathbb{E}\left[D^{-}(1)\right]>0$, respectively. We assumed a finite second moment for $T$, so that the second moment of $D$ is finite, implying $\mathbb{E}[|D(1)|]=\mathbb{E}\left[D^{+}(1)\right]+\mathbb{E}\left[D^{-}(1)\right]<\infty$.

Assume $\mathbb{E}\left[D^{+}(t)\right]=t \mathbb{E}\left[D^{+}(1)\right], t \geq 0$, so that $\mathbb{E}\left[D^{+}(1)\right]=\mathbb{E}\left[(D(n) / n)^{+}\right], n=1,2,3, \ldots$ Consequently, we have $\lim _{n \rightarrow \infty} \mathbb{E}\left[(D(n) / n)^{+}\right]=\mathbb{E}\left[\mathbb{E}[D(1)]^{+}\right]=\mathbb{E}[D(1)]^{+}$, as convergence in mean holds in the context of the strong law of large numbers for independent and identically distributed integrable random variables. This leads to the contradiction $\mathbb{E}\left[D^{+}(1)\right]=\mathbb{E}[D(1)]^{+}=$ 
$\left(\mathbb{E}\left[D^{+}(1)\right]-\mathbb{E}\left[D^{-}(1)\right]\right)^{+}<\mathbb{E}\left[D^{+}(1)\right]$. To summarise, $t \mapsto \mathbb{E}\left[D^{+}(t)\right], t \geq 0$ cannot be a linear function.

On the RHS of (5.9), taking partial derivatives twice with respect to $\boldsymbol{\theta}=\left(\theta_{1}, \theta_{2}\right)$ under the expectation and applying dominated convergence to $\boldsymbol{\theta} \rightarrow \mathbf{0}$, we derive the Wald-type identity

$$
\begin{gathered}
\operatorname{Cov}\left(X_{1}\left(T_{1}(t)\right), X_{2}\left(T_{2}(t)\right)\right)=\mathbb{E}\left[X_{1}(1)\right] \mathbb{E}\left[X_{2}(1)\right] \operatorname{Cov}\left(T_{1}(t), T_{2}(t)\right) \\
+\rho \mathbb{E}\left[T_{1}(t) \wedge T_{2}(t)\right] .
\end{gathered}
$$

By our assumptions, $T$ and $X$ admit finite second moments, so that both sides of (5.10) are finite.

Contradicting the hypothesis, assume $X(T(t)) \stackrel{D}{=} Y(t)$, for all $t \geq 0$, where $Y$ is a given bivariate Lévy process. Plainly, $T$ and $Y$ are Lévy processes with finite second moments. In particular, $t \mapsto \operatorname{Cov}\left(T_{1}(t), T_{2}(t)\right)$ and $t \mapsto \operatorname{Cov}\left(Y_{1}(t), Y_{2}(t)\right)$ are linear functions, and so is $t \mapsto \mathbb{E}\left[T_{1}(t) \wedge T_{2}(t)\right]$, as we assumed $\rho \neq 0$ in (5.10).

Also, $t \mapsto \mathbb{E}\left[T_{2}(t)\right]$ is linear, so that noting $\mathbb{E}\left[T_{1}(t) \wedge T_{2}(t)\right]=\mathbb{E}\left[T_{2}(t)\right]-\mathbb{E}\left[D^{+}(t)\right], t \geq 0$, contradicts the non-linearity of $t \mapsto \mathbb{E}\left[D^{+}(t)\right]$, completing the proof of (i).

(ii) If $T_{1}, T_{2}$ are independent and drift-less, the components of $X \odot T$ are independent by Proposition 3.8. Then using Proposition 3.3 on each component yields $X \odot T \stackrel{D}{=}\left(X_{1} \circ T_{1}, X_{2}^{*} \circ T_{2}\right)$ for independent Lévy processes $T_{1}, T_{2}, X_{1}, X_{2}^{*}$, where $X_{2}^{*} \stackrel{D}{=} X_{2}$. To summarise, we have

$$
\Phi_{X \odot T(t)}(\boldsymbol{\theta})=\mathbb{E}\left[\exp \left\{T_{1}(t) \Psi_{X_{1}}\left(\theta_{1}\right)+T_{2}(t) \Psi_{X_{2}}\left(\theta_{2}\right)\right\}\right], \quad \boldsymbol{\theta}=\left(\theta_{1}, \theta_{2}\right) \in \mathbb{R}^{2} .
$$

Next, note $(r \wedge s) z+(s-r)^{+} z_{2}+(s-r)^{-} z_{1}=(r \wedge s) \widehat{z}+\left(r z_{1}+s z_{2}\right), r, s \geq 0, z, z_{1}, z_{2}, \widehat{z}:=$ $z-z_{1}-z_{2} \in \mathbb{C}$.

As we assume that $X_{1}, X_{2}$ are dependent, there exists $\boldsymbol{\theta}^{*} \in \mathbb{R}^{2}$ such that $\widehat{\Psi}_{X}\left(\boldsymbol{\theta}^{*}\right) \neq 0$. Further, $\Psi_{X_{j}}\left(\theta_{j}^{*}\right) \leq 0, j=1,2, \widehat{\Psi}_{X}\left(\boldsymbol{\theta}^{*}\right) \in \mathbb{R}$ by our symmetry assumption $X \stackrel{D}{=}-X$. If for all $t>0$, (5.11) matches (5.9), we have

$$
\mathbb{E}\left[\exp \left\{T_{1}(t) \Psi_{X_{1}}\left(\theta_{1}^{*}\right)+T_{2}(t) \Psi_{X_{2}}\left(\theta_{2}^{*}\right)\right\}\left(\exp \left\{\left(T_{1}(t) \wedge T_{2}(t)\right) \widehat{\Psi}_{X}\left(\boldsymbol{\theta}^{*}\right)\right\}-1\right)\right]=0,
$$

with the implication $T_{1}(t) \wedge T_{2}(t)=0$, a.s., for all $t>0$. In particular, the null process and $T_{1} \wedge T_{2}$ must be indistinguishable as processes, which is a contradiction to $T_{1}, T_{2}$ being nontrivial subordinators, completing the proof.

\subsection{Proof of Proposition 4.1}

(i) See [7], Part (a) of their Proposition 2.1.

(ii) Let $0<q<1$ and $B \sim B M^{n}(\boldsymbol{\mu}, \Sigma)$. If $\mathbf{t} \in[0, \infty)_{*}^{n}$, set $\psi(\mathbf{t}):=\mathbb{E}\left[\|B(\mathbf{t})\|^{2 q} \mathbf{1}_{\mathbb{D}_{*}}(B(\mathbf{t}))\right]$.

' $\Rightarrow$ ': Note $\psi(\mathbf{t}) \leq 1$ for $\mathbf{t} \in[0, \infty)^{n}$, and introduce $|\Sigma|:=\left(\left|\Sigma_{k l}\right|\right) \in \mathbb{R}^{n \times n},|Z|:=\left(\left|Z_{1}\right|, \ldots\right.$, $\left.\left|Z_{n}\right|\right)$ for a standard normal vector $\left(Z_{1}, \ldots, Z_{n}\right) \in \mathbb{R}^{n}$ and $Q:=2^{(2 q) \vee 1}\left(\|\boldsymbol{\mu}\|^{2 q}+\mathbb{E}\left[\||Z|\|_{|\Sigma|}^{2 q}\right]\right)$. If $\mathbf{t} \in[0, \infty)_{*}^{n}$, we have $\psi(\mathbf{t}) \leq \mathbb{E}\left[\|B(\mathbf{t})\|^{2 q}\right] \leq Q\left(\|\mathbf{t}\|^{q} \vee\|\mathbf{t}\|^{2 q}\right)$ and thus $\psi(\mathbf{t}) \leq(1 \vee Q)\left(1 \wedge\|\mathbf{t}\|^{q}\right)$. As $\mathcal{Y}(\mathrm{d} \mathbf{y})=\mathbb{P}(B(\mathbf{t}) \in \mathrm{d} \mathbf{y}) \mathcal{T}(\mathrm{dt})$ in (3.5), sufficiency follows from this and Part (i).

' $\Leftarrow$ ': Assume an invertible $\Sigma$. Set $\mathbb{D}_{*}^{+}:=\mathbb{D} \cap[0, \infty)_{*}^{n}$. The proof is completed, provided we can show that $i:=\inf _{\mathbf{t} \in \mathbb{D}_{*}^{+}} \psi(\mathbf{t}) /\|\mathbf{t}\|^{q}>0$. If $\mathbf{t} \in[0, \infty)_{*}^{n}$, then set $\phi(\mathbf{t}):=\psi(\mathbf{t}) /\|\mathbf{t}\|^{q}$. Plainly, 
we have $\mathbf{t}_{m} \rightarrow \mathbf{t}_{0}, \mathbf{s}_{m}:=\mathbf{t}_{m} /\left\|\mathbf{t}_{m}\right\| \rightarrow \mathbf{s}_{0}$ and $\phi\left(\mathbf{t}_{m}\right) \rightarrow i$ as $m \rightarrow \infty$ for some $\mathbf{t}_{0} \in \mathbb{D}, \mathbf{s}_{0} \in \mathbb{S}_{+}$, $\mathbf{t}_{m} \in \mathbb{D}_{*}, m \geq 1$.

If $\mathbf{t}_{0} \neq \mathbf{0}$, then we find $\varnothing \neq J \subseteq\{1, \ldots, n\}$ such that $\mathbf{t}_{0} \in C_{J}:=\left\{\sum_{j \in J} x_{j} \mathbf{e}_{j}: x_{j}>0\right.$ for all $j \in J\}$. Note $\mathbf{t}_{0} \diamond \boldsymbol{\mu} \in \mathbb{R}^{n} \boldsymbol{\pi}_{J}$, while $\mathbf{t}_{0} \diamond \Sigma: \mathbb{R}^{n} \boldsymbol{\pi}_{J} \rightarrow \mathbb{R}^{n} \boldsymbol{\pi}_{J}$ is invertible. Particularly, $\mathbb{P}(B(\mathbf{t}) \neq \mathbf{0})=1, \mathbb{P}(0<\|B(\mathbf{t})\|<1)>0$ and $\psi^{*}(\mathbf{t}):=\mathbb{E}\left[\|B(\mathbf{t})\|^{2 q} \mathbf{1}_{(0,1)}(\|B(\mathbf{t})\|)\right]>0$. As desired, we get from Fatou's lemma and the continuity of the sample paths of $B$ that $i=\left\|\mathbf{t}_{0}\right\|^{-q} \liminf _{m \rightarrow \infty} \psi\left(\mathbf{t}_{m}\right) \geq\left\|\mathbf{t}_{0}\right\|^{-q} \psi^{*}\left(\mathbf{t}_{0}\right)>0$.

If $\mathbf{t}_{0}=\mathbf{0}$, let $B^{*} \sim B M^{n}(\mathbf{0}, \Sigma)$, and recall $\left\|\mathbf{t}_{m}\right\|^{-\frac{1}{2}} B\left(\mathbf{t}_{m}\right) \stackrel{D}{=} \boldsymbol{\mu} \diamond\left(\left\|\mathbf{t}_{m}\right\|^{\frac{1}{2}} \mathbf{s}_{m}\right)+B^{*}\left(\mathbf{s}_{m}\right)=: W_{m} \rightarrow$ $B^{*}\left(\mathbf{s}_{0}\right)$ and $\mathbf{1}_{\mathbb{D}_{*}}\left(\|\mathbf{t}\|^{1 / 2} W_{m}\right)=\mathbf{1}_{\mathbb{D}}\left(\|\mathbf{t}\|^{1 / 2} W_{m}\right) \rightarrow 1$, almost surely, as $m \rightarrow \infty$, by continuity of the sample paths of $B^{*}$. The proof of the necessity is completed by Fatou's lemma as

$$
i \geq \liminf _{m \rightarrow \infty} \mathbb{E}\left[\left\|W_{m}\right\|^{2 q} \mathbf{1}_{\mathbb{D}_{*}}\left(\|\mathbf{t}\|^{1 / 2} W_{m}\right)\right] \geq \mathbb{E}\left[\left\|B^{*}\left(\mathbf{s}_{0}\right)\right\|^{2 q}\right]>0 .
$$

(iii) Let $Y \sim V G G^{n}(\mathbf{d}, \boldsymbol{\mu}, \Sigma, \mathcal{U})$. To have $Y \sim F V^{n}$ for an invertible $\Sigma$, we cannot allow for a non-trivial Brownian component in (4.5), thus forcing $\mathbf{d}=\mathbf{0}$. The Lévy measure of an $F V^{n}$. process obeys (2.3), and the necessity part of Proposition 4.1(ii) forces $\int_{\mathbb{D} C} \mathcal{U}(\mathrm{du}) /\|\mathbf{u}\|^{1 / 2}$ to be finite for invertible $\Sigma$. If $\mathbf{d}=\mathbf{0}$ then $Y$ in (4.5) has no Brownian component. If $\int_{\mathbb{D}^{C}} \mathcal{U}(\mathrm{du}) /\|\mathbf{u}\|^{1 / 2}$ is finite, then so is (2.3) for $\mathcal{Y}$, as an implication of Proposition 4.1 for $q=1$. If $\mathbf{d}=\mathbf{0}$ and $\int_{\mathbb{D}^{C}} \mathcal{U}(\mathrm{d} \mathbf{u}) /\|\mathbf{u}\|^{1 / 2}<\infty$ hold simultaneously with no invertibility assumptions on $\Sigma$, then so does $Y \sim F V^{n}$.

\section{Acknowledgements}

We are grateful to two referees for their very careful reading and for suggesting substantial improvements to the original version of the paper. We thank M. Michaelsen, C. Rau and A. Szimayer for their comments on an earlier version of the manuscript. B. Buchmann's research was partially supported by ARC grant DP160104737. K. Lu's research was supported by an Australian Government Research Training Program Scholarship.

\section{References}

[1] Applebaum, D. (2009). Lévy Processes and Stochastic Calculus, 2nd ed. Cambridge Studies in Advanced Mathematics 116. Cambridge: Cambridge Univ. Press. MR2512800

[2] Barndorff-Nielsen, O.E., Maejima, M. and Sato, K. (2006). Some classes of multivariate infinitely divisible distributions admitting stochastic integral representations. Bernoulli 12 1-33. MR2202318

[3] Barndorff-Nielsen, O.E., Pedersen, J. and Sato, K. (2001). Multivariate subordination, selfdecomposability and stability. Adv. in Appl. Probab. 33 160-187. MR1825321

[4] Barndorff-Nielsen, O.E. and Shiryaev, A. (2010). Change of Time and Change of Measure. Advanced Series on Statistical Science \& Applied Probability 13. Hackensack, NJ: World Scientific. MR2779876

[5] Bertoin, J. (1996). Lévy Processes. Cambridge: Cambridge Univ. Press. MR1406564

[6] Bondesson, L. (2009). On univariate and bivariate generalized gamma convolutions. J. Statist. Plann. Inference 139 3759-3765. MR2553760 
[7] Buchmann, B., Kaehler, B., Maller, R. and Szimayer, A. (2017). Multivariate subordination using generalised gamma convolutions with applications to variance gamma processes and option pricing. Stochastic Process. Appl. 127 2208-2242. MR3652411

[8] Buchmann, B., Lu, K.W. and Madan, D.B. (2017). Parameter estimation for weak variance-alphagamma processes. Australian National University, University of Maryland. Preprint.

[9] Buchmann, B., Lu, K.W. and Madan, D.B. (2017). Self-decomposability of variance generalised gamma convolutions. Australian National University, University of Maryland. Preprint.

[10] Gradshteyn, I.S. and Ryzhik, I.M. (1996). Table of Integrals, Series, and Products, 5th ed. San Diego, CA: Academic Press. MR1398882

[11] Grigelionis, B. (2007). On subordinated multivariate Gaussian Lévy processes. Acta Appl. Math. 96 233-246. MR2327538

[12] Guillaume, F. (2013). The $\alpha$ VG model for multivariate asset pricing: Calibration and extension. Rev. Deriv. Res. 16 25-52.

[13] James, L.F., Roynette, B. and Yor, M. (2008). Generalized gamma convolutions, Dirichlet means, Thorin measures, with explicit examples. Probab. Surv. 5 346-415. MR2476736

[14] Luciano, E. and Semeraro, P. (2010). Multivariate time changes for Lévy asset models: Characterization and calibration. J. Comput. Appl. Math. 233 1937-1953. MR2564029

[15] Madan, D.B. (2017). Instantaneous Portfolio Theory. University of Maryland. Preprint. Available at https://papers.ssrn.com/sol3/papers.cfm?abstract_id=2804718.

[16] Madan, D.B. and Seneta, E. (1990). The variance gamma (v.g.). model for share market returns. J. Bus. 63 511-524.

[17] Michaelsen, M. (2017). Information flow dependence in financial markets. Universität Hamburg. Preprint.

[18] Michaelsen, M. and Szimayer, A. (2017). Marginal consistent dependence modeling using weak subordination for Brownian motions. Universität Hamburg. Preprint.

[19] Pérez-Abreu, V. and Stelzer, R. (2014). Infinitely divisible multivariate and matrix gamma distributions. J. Multivariate Anal. 130 155-175. MR3229530

[20] Rogozin, B.A. (1965). On some classes of processes with independent increments. Theory Probab. Appl. 10 479-483. MR0189124

[21] Sato, K. (1999). Lévy Processes and Infinitely Divisible Distributions. Cambridge Studies in Advanced Mathematics 68. Cambridge: Cambridge Univ. Press. MR1739520

[22] Schilling, R.L., Song, R. and Vondraček, Z. (2010). Bernstein Functions. Berlin: Walter de Gruyter. MR2978140

[23] Semeraro, P. (2008). A multivariate variance gamma model for financial applications. Int. J. Theor. Appl. Finance 11 1-18. MR2398464

[24] Thorin, O. (1977). On the infinite divisibility of the Pareto distribution. Scand. Actuar. J. 1 31-40. MR0431333

[25] Thorin, O. (1977). On the infinite divisibility of the lognormal distribution. Scand. Actuar. J. 1977 121-148. MR0552135

[26] Zolotarev, V.M. (1958). Distribution of the superposition of infinitely divisible processes (Russian). Teor. Verojatnost. i Primenen. 3 197-200. MR0123355

Received June 2017 and revised October 2017 